\title{
Spectral discretization of the time-dependent Navier-Stokes problem coupled with the heat equation
}

\author{
Rahma Agroum ${ }^{1}$, Christine Bernardi ${ }^{2}$, and Jamil Satouri ${ }^{3}$
}

\begin{abstract}
The aim of this work is to present the unsteady Navier-Stokes equations coupled with the heat equation where the viscosity depends on the temperature. We propose a discretization of theses equations that combines Euler's implicit scheme in time and spectral methods in space. We prove optimal error estimates between the continuous and discrete solutions. Some numerical experiments confirm the interest of this approach.
\end{abstract}

\section{Résumé}

On présente dans ce travail les équations de Navier-Stokes instationnaires couplées avec celle de la chaleur lorsque la viscosité dépend de la température. On utilise un shéma d'Euler implicite en temps et on discrétise le problème en espace par méthodes spectrales. On démontre des estimations d'erreur optimales entre la solution continue et la solution discrète. Quelques expériences numériques confirment l'intérêt de cette approche.

Key words: Navier-Stokes equations, heat equation, spectral method, Euler's scheme, a priori analysis.

\footnotetext{
${ }^{1}$ Presently at Laboratoire Jacques-Louis Lions, Université Pierre et Marie Curie, 4 place Jussieu, 75252 Paris cedex 05, France. email-address: agroum@ann.jussieu.fr

${ }^{2}$ CNRS, UMR 7598, Laboratoire Jacques-Louis Lions, F-75005, Paris, France Sorbonne Universités, UPMC Univ Paris 06, UMR 7598, Laboratoire Jacques-Louis Lions, F-75005, Paris, France email-address: bernardi@ann.jussieu.fr

${ }^{3}$ IPEIT-University of Tunis, 2 rue Jawaher Lel Nahra-1089, Monfleury, Tunisia. e-mail address: satouri@yahoo.fr
} 



\section{Introduction.}

Let $\Omega$ be a connected bounded open set in $\mathbb{R}^{d}, d=2$ or 3 , with a Lipschitz-continuous boundary $\partial \Omega$, and let $[0, \widetilde{T}]$ denote an interval in $\mathbb{R}$ where $\widetilde{T}$ is a positive real number. The following system models the unsteady flow of a viscous incompressible fluid, in the case where the viscosity of the fluid depends on the temperature.

$$
\begin{array}{lr}
\partial_{t} \boldsymbol{u}(\boldsymbol{x}, t)-\operatorname{div}(\nu(T(x, t)) \nabla \boldsymbol{u}(\boldsymbol{x}, t))+(\boldsymbol{u}(\boldsymbol{x}, t) \cdot \nabla) \boldsymbol{u}(\boldsymbol{x}, t)+\nabla p(\boldsymbol{x}, t)=\boldsymbol{f}(\boldsymbol{x}, t) & \text { in } \Omega \times] 0, \widetilde{T}[, \\
\operatorname{div} \boldsymbol{u}(\boldsymbol{x}, t)=0 & \text { in } \Omega \times] 0, \widetilde{T}[, \\
\partial_{t} T(\boldsymbol{x}, t)-\alpha \Delta T(\boldsymbol{x}, t)+(\boldsymbol{u}(\boldsymbol{x}, t) \cdot \nabla) T(\boldsymbol{x}, t)=g(\boldsymbol{x}, t) & \text { in } \Omega \times] 0, \widetilde{T}[, \\
T(\boldsymbol{x}, t)=T_{b} & \text { on } \partial \Omega \times] 0, \widetilde{T}[, \\
\boldsymbol{u}(\boldsymbol{x}, t)=\boldsymbol{u}_{D} & \text { on } \partial \Omega \times] 0, \widetilde{T}[, \\
\boldsymbol{u}(\boldsymbol{x}, 0)=\boldsymbol{u}_{0}, \quad T(\boldsymbol{x}, 0)=T_{0} & \text { in } \Omega .
\end{array}
$$

The unknowns are the velocity $\boldsymbol{u}$, the pressure $p$, and the temperature $T$ of the fluid, while the body data are the distributions $\boldsymbol{f}$ and $g$, the boundary data are the Dirichlet condition $\boldsymbol{u}_{D}$, and the temperature on the boundary $T_{b}$, the initial data are $\boldsymbol{u}_{0}$ and the initial value of the temperature $T_{0}$ in $\Omega$. The function $\nu$ is positive and bounded, while the coefficient $\alpha$ is a positive constant.

A similar model but for the stationary case has been analyzed in [8] and recently in [1] for a spectral discretization and also in [4] for a finite element discretization (see also [3] for a very similar coupling of the Navier-Stokes equations with a turbulence model). Another related model has been analyzed in [2]: it consists of the heat equation with a nonlinear source term describing heat production due to an exothermic chemical reaction coupled with the Darcy law.

Problem (1.1) is discretized in time by Euler's implicit scheme. We use this scheme for its simplicity, however we have decided to treat the nonlinear term in an implicit way; we combine this scheme with a spectral method in space. The numerical analysis of the nonlinear discrete problem makes use of the approach of Brezzi, Rappaz and Raviart [7], we establish a priori error estimates that turn out to be fully optimal. We conclude with some numerical experiments where the viscosity of the fluid $\nu$ is either a constant, or a function dependent of the space variable, or a function that depends on the temperature $T$. All of them confirm the optimality of the discretization and justify the choice of this formulation.

An outline of the paper is as follows.

- In Section 2, we recall the variational formulation of the problem and prove the existence of a solution, the uniqueness result being presented in dimension $d=2$.

- Section 3 is devoted to the description of the time semi-discrete problem, we recall the existence of the solution. We also write the fully discrete problem.

- In Section 4, we perform the a priori error analysis of the discretization.

- Some numerical experiments are presented in Section 5. 


\section{Analysis of the continuous problem.}

We first write down a variational formulation of problem (1.1). Next, we prove the existence of the solution. The uniqueness is only proved in dimension $d=2$.

\subsection{Variational formulation}

We intend to write a variational formulation of system (1.1). We first make precise the assumptions on the function $\nu$ : It belongs to $L^{\infty}(\mathbb{R})$ and satisfies, for two positive constants $\nu_{1}$ and $\nu_{2}$,

$$
\text { for a.e. } \theta \in \mathbb{R}, \quad \nu_{1} \leqslant \nu(\theta) \leqslant \nu_{2} \text {. }
$$

For simplicity, we work with zero bondary condition $\boldsymbol{u}_{D}=\mathbf{0}$ in order to avoid the technical results linked to the Hopf lemma, see [11, Chap. IV, Thm 2.3].

In what follows, the scalar product defined on $L^{2}(\Omega)$ or $L^{2}(\Omega)^{d}$ is denoted by $(.,$.$) . We use the whole$ scale of Sobolev spaces $H^{s}(\Omega), s \geqslant 0$, equipped with the norm $\|\cdot\|_{H^{s}(\Omega)}$ and seminorm $|\cdot|_{H^{s}(\Omega)}$, and their subspaces $H_{0}^{s}(\Omega)$. For each $s \geqslant 0, H^{-s}(\Omega)$ stands for the dual space of $H_{0}^{s}(\Omega)$. We also recall from [11, Chap. I, Thm 1.1] for instance the following Poincaré-Friedrichs inequality:

$$
\forall \varphi \in H_{0}^{1}(\Omega), \quad\|\varphi\|_{L^{2}(\Omega)} \leqslant c|\varphi|_{H^{1}(\Omega)},
$$

which yields the equivalence of $\|\cdot\|_{H^{1}(\Omega)}$ and $|\cdot|_{H^{1}(\Omega)}$ on $H_{0}^{1}(\Omega)$.

For any separable Banach space $E$ equipped with the norm $\|.\|_{E}$, we denote by $\mathscr{C}^{0}(0, \widetilde{T} ; E)$ the space of continuous functions from $[0, \widetilde{T}]$ with values in $E$. For each $s \geqslant 0$, we also introduce the space $H^{s}(0, \widetilde{T} ; E)$ in the following way: When $s$ is an integer, it is the space of measurable functions on $] 0, \widetilde{T}[$ with values in $E$ such that the mappings: $\boldsymbol{v} \mapsto\left\|\partial_{t}^{\ell} \boldsymbol{v}\right\|_{E}, 0 \leqslant \ell \leqslant s$, are square-integrable on $] 0, \widetilde{T}[$; otherwise, it is defined by interpolation between $H^{[s]+1}(0, \widetilde{T} ; E)$ and $H^{[s]}(0, \widetilde{T} ; E)$, where $[s]$ stands for the integer part of $s$.

We also introduce the space

$$
L_{\circ}^{2}(\Omega)=\left\{q \in L^{2}(\Omega) ; \int_{\Omega} q(\boldsymbol{x}) \mathrm{d} \boldsymbol{x}=0\right\} .
$$

Assume that the data $\left(\boldsymbol{f}, g, T_{b}\right)$ belong to

$$
L^{2}\left(0, \widetilde{T} ; H^{-1}(\Omega)^{d}\right) \times L^{2}\left(0, \widetilde{T} ; H^{-1}(\Omega)\right) \times L^{2}\left(0, \widetilde{T} ; H^{\frac{1}{2}}(\partial \Omega)\right),
$$

that the datum $T_{0}$ belongs to $H^{1}(\Omega)$, the datum $\boldsymbol{u}_{0}$ belongs to $H_{0}^{1}(\Omega)^{d}$ and satisfies the following compatibility condition

$$
\operatorname{div} \boldsymbol{u}_{0}=0 \quad \text { in } \Omega \text {. }
$$

The last condition is not necessary for all the results in this section. Since it is not restrictive, we prefer to assume it from now on.

We now consider the following variational formulation for problem (1.1):

Find $(\boldsymbol{u}, p, T)$ in $L^{2}\left(0, \widetilde{T} ; H_{0}^{1}(\Omega)^{d}\right) \times L^{2}\left(0, \widetilde{T} ; L_{\circ}^{2}(\Omega)\right) \times L^{2}\left(0, \widetilde{T} ; H^{1}(\Omega)\right)$ such that

$$
\boldsymbol{u}(., 0)=\boldsymbol{u}_{0} \quad \text { and } \quad T(., 0)=T_{0} \quad \text { in } \Omega,
$$

such that, for a.e. $t, 0 \leqslant t \leqslant \widetilde{T}$,

$$
T(., t)=T_{b}(., t) \quad \text { on } \partial \Omega
$$


and such that, for a.e. $t, 0 \leqslant t \leqslant \widetilde{T}$,

$$
\begin{gathered}
\forall \boldsymbol{v} \in H_{0}^{1}(\Omega)^{d}, \quad \int_{\Omega} \partial_{t} \boldsymbol{u}(\boldsymbol{x}, t) \boldsymbol{v}(\boldsymbol{x}) \mathrm{d} \boldsymbol{x}+\int_{\Omega} \nu(T)(\boldsymbol{x}, t)(\operatorname{grad} \boldsymbol{u})(\boldsymbol{x}, t):(\operatorname{grad} \boldsymbol{v})(\boldsymbol{x}) \mathrm{d} \boldsymbol{x} \\
+\int_{\Omega}((\boldsymbol{u} \cdot \nabla) \boldsymbol{u})(\boldsymbol{x}, t) \cdot \boldsymbol{v}(\boldsymbol{x}) \mathrm{d} \boldsymbol{x}-\int_{\Omega}(\operatorname{div} \boldsymbol{v})(\boldsymbol{x}) p(\boldsymbol{x}, t) \mathrm{d} \boldsymbol{x}=\langle\boldsymbol{f}, \boldsymbol{v}\rangle_{\Omega}, \\
\begin{aligned}
\forall q \in L_{\circ}^{2}(\Omega), \quad-\int_{\Omega}(\operatorname{div} \boldsymbol{u})(\boldsymbol{x}, t) q(\boldsymbol{x}) \mathrm{d} \boldsymbol{x}=0, \\
\forall S \in H_{0}^{1}(\Omega), \quad \int_{\Omega} \partial_{t} T(\boldsymbol{x}, t) S(\boldsymbol{x}) \mathrm{d} \boldsymbol{x}+\alpha \int_{\Omega}(\operatorname{grad} T)(\boldsymbol{x}, t) \cdot(\operatorname{grad} S)(\boldsymbol{x}) \mathrm{d} \boldsymbol{x} \\
+\int_{\Omega}((\boldsymbol{u} \cdot \nabla) T)(\boldsymbol{x}, t) S(\boldsymbol{x}) \mathrm{d} \boldsymbol{x}=\langle g, S\rangle_{\Omega},
\end{aligned}
\end{gathered}
$$

where $\langle., .\rangle_{\Omega}$ denotes the duality pairing between $H^{-1}(\Omega)$ and $H_{0}^{1}(\Omega)$ and also between $H^{-1}(\Omega)^{d}$ and $H_{0}^{1}(\Omega)^{d}$. The following result is an obvious consequence of the density of $\mathscr{D}(\Omega)$ in $L^{2}(\Omega)$ or $H_{0}^{1}(\Omega)$.

Proposition 2.1 Problem (1.1) with $\boldsymbol{u}_{D}=0$ and (2.5)-(2.6)-(2.7) are equivalent, in the sense that any pair $(\boldsymbol{u}, p, T)$ in $L^{2}\left(0, \widetilde{T} ; H_{0}^{1}(\Omega)^{d}\right) \times L^{2}\left(0, \widetilde{T} ; L_{\circ}^{2}(\Omega)\right) \times L^{2}\left(0, \widetilde{T} ; H^{1}(\Omega)\right)$ is a solution of system (1.1) in the distribution sense if and only if it is a solution of problem $(2.5)-(2.6)-(2.7)$.

The spaces $L_{\circ}^{2}(\Omega)$ and $H_{0}^{1}(\Omega)^{d}$ verify the inf-sup condition (see for instance [11, Chap. I, Cor. 2.4]): There exist a constant $\beta>0$ such that

$$
\forall q \in L_{\circ}^{2}(\Omega), \quad \sup _{\boldsymbol{v} \in H_{0}^{1}(\Omega)^{d}} \frac{\int_{\Omega}(\operatorname{div} \boldsymbol{v})(\boldsymbol{x}) q(\boldsymbol{x}) \mathrm{d} \boldsymbol{x}}{\|\boldsymbol{v}\|_{H^{1}(\Omega)^{d}}} \geqslant \beta\|q\|_{L^{2}(\Omega)} .
$$

We introduce the kernel

$$
V(\Omega)=\left\{\boldsymbol{v} \in H_{0}^{1}(\Omega)^{d} ; \forall q \in L_{\circ}^{2}(\Omega), \int_{\Omega}(\operatorname{div} \boldsymbol{v})(\boldsymbol{x}) q(\boldsymbol{x}) \mathrm{d} \boldsymbol{x}=0\right\},
$$

which is a closed subspace of $H_{0}^{1}(\Omega)^{d}$ and coincides with

$$
V(\Omega)=\left\{\boldsymbol{v} \in H_{0}^{1}(\Omega)^{d} ; \operatorname{div} \boldsymbol{v}=0 \text { in } \Omega\right\} .
$$

Let $\mathcal{R}$ denote a lifting operator, i.e., an operator from $H^{\frac{1}{2}}(\partial \Omega)$ into $H^{1}(\Omega)$ which is continuous from $H^{s+\frac{1}{2}}(\partial \Omega)$ into $H^{s+1}(\Omega)$ for all $s \geqslant 0$ (the existence of such an operator is established in [9] for instance). Since $T_{b}$ belongs to $L^{2}\left(0, \widetilde{T} ; H^{\frac{1}{2}}(\partial \Omega)\right)$ we denote by $\overline{T_{b}}$ the function defined for a.e. $t$, $0 \leqslant t \leqslant \widetilde{T}$, by

$$
\overline{T_{b}}(t)=\mathcal{R} T_{b}(t) .
$$

Clearly this function belongs to $L^{2}\left(0, \widetilde{T} ; H^{1}(\Omega)\right)$ and satisfies

$$
\left\|\overline{T_{b}}\right\|_{L^{2}\left(0, \widetilde{T} ; H^{1}(\Omega)\right)} \leqslant c_{\diamond}\left\|T_{b}\right\|_{L^{2}\left(0, \widetilde{T} ; H^{\frac{1}{2}}(\partial \Omega)\right)}
$$

where the positive constant $c$ only depends on $\Omega$ and $\mathcal{R}$. When setting $T^{*}=T-\overline{T_{b}}$, we observe that $\left(\boldsymbol{u}, p, T^{*}\right)$ is a solution of the variational problem:

Find $\left(\boldsymbol{u}, p, T^{*}\right)$ in $L^{2}\left(0, \widetilde{T} ; H_{0}^{1}(\Omega)^{d}\right) \times L^{2}\left(0, \widetilde{T} ; L_{\circ}^{2}(\Omega)\right) \times L^{2}\left(0, \widetilde{T} ; H_{0}^{1}(\Omega)\right)$ such that

$$
\boldsymbol{u}(., 0)=\boldsymbol{u}_{0} \quad \text { and } \quad T^{*}(., 0)=T_{0}-\overline{T_{b}}(., 0) \quad \text { in } \Omega,
$$


and such that, for a.e. $t, 0 \leqslant t \leqslant \widetilde{T}$,

$$
\begin{aligned}
& \forall \boldsymbol{v} \in H_{0}^{1}(\Omega)^{d}, \quad\left(\partial_{t} \boldsymbol{u}, \boldsymbol{v}\right)+\left(\nu\left(T^{*}+\overline{T_{b}}\right) \nabla \boldsymbol{u}, \nabla \boldsymbol{v}\right)+((\boldsymbol{u} . \nabla) \boldsymbol{u}, \boldsymbol{v})-(\operatorname{div} \boldsymbol{v}, p)=\langle\boldsymbol{f}, \boldsymbol{v}\rangle_{\Omega}, \\
& \forall q \in L_{\circ}^{2}(\Omega), \quad-(\operatorname{div} \boldsymbol{u}, q)=0, \\
& \forall S \in H_{0}^{1}(\Omega), \quad\left(\partial_{t} T^{*}, S\right)+\alpha\left(\nabla T^{*}, \nabla S\right)+\left((\boldsymbol{u} . \nabla) T^{*}, S\right) \\
& =\langle g, S\rangle_{\Omega}-\left(\partial_{t} \overline{T_{b}}, S\right)-\alpha\left(\nabla \overline{T_{b}}, \nabla S\right)-\left((\boldsymbol{u} \cdot \nabla) \overline{T_{b}}, S\right) .
\end{aligned}
$$

Moreover, the pair $\left(\boldsymbol{u}, T^{*}\right)$ is a solution of the variational problem:

Find $\left(\boldsymbol{u}, T^{*}\right)$ in $L^{2}(0, \widetilde{T} ; V(\Omega)) \times L^{2}\left(0, \widetilde{T} ; H_{0}^{1}(\Omega)\right)$ satisfying $(2.10)$ and, for a.e. $t, 0 \leqslant t \leqslant \widetilde{T}$,

$$
\begin{aligned}
& \forall \boldsymbol{v} \in V(\Omega), \quad\left(\partial_{t} \boldsymbol{u}, \boldsymbol{v}\right)+\left(\nu\left(T^{*}+\overline{T_{b}}\right) \nabla \boldsymbol{u}, \nabla \boldsymbol{v}\right)+((\boldsymbol{u} . \nabla) \boldsymbol{u}, \boldsymbol{v})=\langle\boldsymbol{f}, \boldsymbol{v}\rangle_{\Omega}, \\
& \forall S \in H_{0}^{1}(\Omega), \quad\left(\partial_{t} T^{*}, S\right)+\alpha\left(\nabla T^{*}, \nabla S\right)+\left((\boldsymbol{u} \cdot \nabla) T^{*}, S\right) \\
& \quad=\langle g, S\rangle_{\Omega}-\left(\partial_{t} \overline{T_{b}}, S\right)-\alpha\left(\nabla \overline{T_{b}}, \nabla S\right)-\left((\boldsymbol{u} . \nabla) \overline{T_{b}}, S\right) .
\end{aligned}
$$

\section{$2.2 \quad$ Existence result}

Let us start with the following a priori estimate for the velocity.

Lemma 2.2 Assume that the data $\boldsymbol{f}$ belong to $L^{2}\left(0, \widetilde{T} ; H^{-1}(\Omega)^{d}\right)$, and that the initial velocity $\boldsymbol{u}_{0}$ belongs to $L^{2}(\Omega)^{d}$. Then the following a priori estimate holds for the velocity $\boldsymbol{u}$ of any solution $\left(\boldsymbol{u}, T^{*}\right)$ of problem (2.10)-(2.12) and for any $t \in] 0, \widetilde{T}[$ :

$$
\|\boldsymbol{u}\|_{L^{2}\left(0, t ; H^{1}(\Omega)^{d}\right)} \leqslant c\left(\left\|\boldsymbol{u}_{0}\right\|_{L^{2}(\Omega)^{d}}+\|\boldsymbol{f}\|_{L^{2}\left(0, t ; H^{-1}(\Omega)^{d}\right)}\right),
$$

with a constant $c$ that only depends on $\Omega$ and $\widetilde{T}$.

Proof: Taking $\boldsymbol{v}$ equal to $\boldsymbol{u}$ in the first equation of (2.12) and noting that $((\boldsymbol{u} . \nabla) \boldsymbol{u}, \boldsymbol{u})=0$ gives

$$
\frac{1}{2} \frac{d}{d t}\|\boldsymbol{u}\|_{L^{2}(\Omega)^{d}}^{2}+\nu_{1}\|\nabla \boldsymbol{u}\|_{L^{2}(\Omega)^{d \times d}}^{2} \leqslant c\|\boldsymbol{f}\|_{H^{-1}(\Omega)^{d}}\|\boldsymbol{u}\|_{H^{1}(\Omega)^{d}}
$$

whence

$$
\frac{d}{d t}\|\boldsymbol{u}\|_{L^{2}(\Omega)^{d}}^{2}+\nu_{1} c\|\boldsymbol{u}\|_{H^{1}(\Omega)^{d}}^{2} \leqslant \frac{c^{\prime}}{\nu_{1}}\|\boldsymbol{f}\|_{H^{-1}(\Omega)^{d}}^{2} .
$$

Integrating this equation between 0 and $t$ yields in particular

$$
\|\boldsymbol{u}(., t)\|_{L^{2}(\Omega)^{d}}^{2}+\nu_{1} c \int_{0}^{t}\|\boldsymbol{u}(., s)\|_{H^{1}(\Omega)^{d}}^{2} d s \leqslant\|\boldsymbol{u}(., 0)\|_{L^{2}(\Omega)^{d}}^{2}+\frac{c^{\prime}}{\nu_{1}} \int_{0}^{t}\|\boldsymbol{f}(., s)\|_{H^{-1}(\Omega)^{d}}^{2} d s
$$

Owing to (2.10), this gives the desired estimate.

Theorem 2.3 Assume that the data $\left(\boldsymbol{f}, g, T_{b}\right)$ belong to $L^{2}\left(0, \widetilde{T} ; H^{-1}(\Omega)^{d}\right) \times L^{2}\left(0, \widetilde{T} ; H^{-1}(\Omega)\right) \times$ $H^{1}\left(0, \widetilde{T} ; H^{\frac{1}{2}}(\partial \Omega)\right)$, that the initial velocity $\boldsymbol{u}_{0}$ belongs to $L^{2}(\Omega)^{d}$, the initial temperature $T_{0}$ belongs to $L^{2}(\Omega)$ and that the initial temperature on the boundary $T_{b}^{0}$ belongs to $H^{\frac{1}{2}}(\partial \Omega)$. Then problem (2.5)-(2.6)-(2.7) has a solution $(\boldsymbol{u}, p, T)$ in the space

$$
L^{2}\left(0, \widetilde{T} ; H_{0}^{1}(\Omega)^{d}\right) \times L^{2}\left(0, \widetilde{T} ; L_{\circ}^{2}(\Omega)\right) \times L^{2}\left(0, \widetilde{T} ; H^{1}(\Omega)\right) .
$$

Proof: 1) We recall from [11, Chap. I, Cor 2.5] that $\mathscr{D}(\Omega)^{d} \cap V(\Omega)$ is dense in $V(\Omega)$. Thus, there exist an increasing sequence $\left(\mathbb{V}_{n}\right)_{n}$ of finite-dimensional subspaces of $V(\Omega)$ and an increasing sequence $\left(\mathbb{W}_{n}\right)_{n}$ of finite-dimensional subspaces of $H_{0}^{1}(\Omega)$ such that $\underset{n \in \mathbb{N}}{\cup}\left(\mathbb{V}_{n} \times \mathbb{W}_{n}\right)$ is dense in $V(\Omega) \times H_{0}^{1}(\Omega)$. 
Thus, an approximation of problem (2.12) can be written Find $\left(\boldsymbol{u}_{n}, T_{n}^{*}\right)$ in $L^{2}\left(0, \widetilde{T} ; \mathbb{V}_{n}\right) \times L^{2}\left(0, \widetilde{T} ; \mathbb{W}_{n}\right)$ such that

$$
\begin{array}{r}
\forall \boldsymbol{v}_{n} \in \mathbb{V}_{n}, \quad\left(\partial_{t} \boldsymbol{u}_{n}, \boldsymbol{v}_{n}\right)+\left(\nu\left(T_{n}^{*}+\overline{T_{b}}\right) \nabla \boldsymbol{u}_{n}, \nabla \boldsymbol{v}_{n}\right)+\left(\left(\boldsymbol{u}_{n} \cdot \nabla\right) \boldsymbol{u}_{n}, \boldsymbol{v}_{n}\right)=\left\langle\boldsymbol{f}, \boldsymbol{v}_{n}\right\rangle_{\Omega}, \\
\forall S_{n} \in \mathbb{W}_{n}, \quad\left(\partial_{t} T_{n}^{*}, S_{n}\right)+\alpha\left(\nabla T_{n}^{*}, \nabla S_{n}\right)+\left(\left(\boldsymbol{u}_{n} \cdot \nabla\right) T_{n}^{*}, S_{n}\right) \\
\quad=\left\langle g, S_{n}\right\rangle_{\Omega}-\left(\partial_{t} \overline{T_{b}}, S_{n}\right)-\alpha\left(\nabla \overline{T_{b}}, \nabla S_{n}\right)-\left(\left(\boldsymbol{u}_{n} \cdot \nabla\right) \overline{T_{b}}, S_{n}\right) .
\end{array}
$$

Considering existence, note that the mapping:

$$
(\boldsymbol{w}, z) \mapsto\left(\begin{array}{c}
\boldsymbol{f}+\operatorname{div}\left(\nu\left(z+\overline{T_{b}}\right) \nabla \boldsymbol{w}\right)-(\boldsymbol{w} \cdot \nabla) \boldsymbol{w} \\
g-\partial_{t} \bar{T}_{b}+\alpha \Delta \overline{T_{b}}-(\boldsymbol{w} \cdot \nabla) \overline{T_{b}}-(\boldsymbol{w} \cdot \nabla) z+\alpha \Delta z
\end{array}\right),
$$

is Lipschitz-continuous on $H^{1}(\Omega)^{d} \times H^{1}(\Omega)$. Therefore it follows from the Cauchy-Lipschitz theorem, see [12, Section 21], that $(2.15)$ has a unique solution $\left(\boldsymbol{u}_{n}, T_{n}^{*}\right)$ in $\mathscr{C}^{0}\left(0, \widetilde{T} ; \mathbb{V}_{n}\right) \times \mathscr{C}^{0}\left(0, \widetilde{T} ; \mathbb{W}_{n}\right)$. Since the norm of $\boldsymbol{u}_{n}$ in $L^{2}\left(0, \widetilde{T} ; H_{0}^{1}(\Omega)^{d}\right)$ and of $T_{n}^{*}$ in $L^{2}\left(0, \widetilde{T} ; H_{0}^{1}(\Omega)\right.$ ) are bounded by a constant $c$ (due to the Poincaré-Friedrichs inequality on $\Omega$ ), there exists a subsequence, still denoted by $\left(\boldsymbol{u}_{n}, T_{n}^{*}\right)_{n}$ for simplicity, which converges to a pair $\left(\boldsymbol{u}, T^{*}\right)$ weakly in $\left.\left.L^{2}\left(0, \widetilde{T} ; H^{1}(\Omega)^{d}\right)\right) \times L^{2}\left(0, \widetilde{T} ; H^{1}(\Omega)\right)\right)$.

We observe that, for $m \leqslant n,\left(\boldsymbol{u}_{n}, T_{n}^{*}\right)$ is a solution of the variational problem:

Find $\left(\boldsymbol{u}_{n}, T_{n}^{*}\right)$ in $L^{2}\left(0, \widetilde{T} ; \mathbb{V}_{n}\right) \times L^{2}\left(0, \widetilde{T} ; \mathbb{W}_{n}\right)$ such that

$$
\begin{aligned}
\forall \boldsymbol{v}_{m} \in \mathbb{V}_{m}, \quad\left(\partial_{t} \boldsymbol{u}_{n}, \boldsymbol{v}_{m}\right)+\left(\nu\left(T_{n}^{*}+\overline{T_{b}}\right) \nabla \boldsymbol{u}_{n}, \nabla \boldsymbol{v}_{m}\right)+\left(\left(\boldsymbol{u}_{n} \cdot \nabla\right) \boldsymbol{u}_{n}, \boldsymbol{v}_{m}\right)=\left\langle\boldsymbol{f}, \boldsymbol{v}_{m}\right\rangle_{\Omega}, \\
\forall S_{m} \in \mathbb{W}_{m}, \quad\left(\partial_{t} T_{n}^{*}, S_{m}\right)+\alpha\left(\nabla T_{n}^{*}, \nabla S_{m}\right)+\left(\left(\boldsymbol{u}_{n} \cdot \nabla\right) T_{n}^{*}, S_{m}\right) \\
=\left\langle g, S_{m}\right\rangle_{\Omega}-\left(\partial_{t} \overline{T_{b}}, S_{m}\right)-\alpha\left(\nabla \overline{T_{b}}, \nabla S_{m}\right)-\left(\left(\boldsymbol{u}_{n} \cdot \nabla\right) \overline{T_{b}}, S_{m}\right) .
\end{aligned}
$$

We now study the convergence on $n$ :

- The convergence of the linear terms is obvious.

- By taking $S_{m}$ equal to $\partial_{t} T_{n}^{*}$ in $(2.16)$, we easily derive that $\partial_{t} T_{n}^{*}$ is bounded in $L^{2}\left(0, \widetilde{T} ; L^{2}(\Omega)\right)$. Due to the compactness of the imbedding of $H^{1}\left(0, \widetilde{T} ; L^{2}(\Omega)\right) \cap L^{2}\left(0, \widetilde{T} ; H^{1}(\Omega)\right)$ into $L^{2}\left(0, \widetilde{T} ; L^{2}(\Omega)\right)$, there exists another subsequence $\left(T_{n}^{*}\right)_{n}$ such that the sequence $\left(\nu\left(T_{n}^{*}+\overline{T_{b}}\right) \nabla \boldsymbol{v}_{m}\right)_{n}$ converges to $\nu\left(T^{*}+\overline{T_{b}}\right) \nabla \boldsymbol{v}_{m}$ a.e. in $\Omega$. Since the norm of $\left(\nu\left(T_{n}^{*}+\overline{T_{b}}\right) \nabla \boldsymbol{v}_{m}\right)_{n}$ is bounded by $\nu_{2}\left\|\nabla \boldsymbol{v}_{m}\right\|_{L^{2}(\Omega)^{d \times d}}$, using the Lebesgue dominated convergence theorem yields the convergence of $\left(\nu\left(T_{n}^{*}+\overline{T_{b}}\right) \nabla \boldsymbol{v}_{m}\right)_{n}$ to $\nu\left(T^{*}+\overline{T_{b}}\right) \nabla \boldsymbol{v}_{m}$ in $L^{2}(\Omega)^{d \times d}$.

- The convergence of the convection terms $\left(\boldsymbol{u}_{n} \cdot \nabla\right) \boldsymbol{u}_{n}$ and $\left(\boldsymbol{u}_{n} \cdot \nabla\right) T_{n}^{*}$ is more complex. So, for brevity we refer to [13, Chap. III, Lemma 3.2] for its proof (see also [10, Chap. V, Thm 1.4]).

Thus, passing to the limit on $n$, problem (2.16) leads to :

Find $\left(\boldsymbol{u}, T^{*}\right)$ in $L^{2}(0, \widetilde{T} ; V(\Omega)) \times L^{2}\left(0, \widetilde{T} ; H_{0}^{1}(\Omega)\right)$ such that

$$
\begin{array}{r}
\forall \boldsymbol{v}_{m} \in \mathbb{V}_{m}, \quad\left(\partial_{t} \boldsymbol{u}, \boldsymbol{v}_{m}\right)+\left(\nu\left(T^{*}+\overline{T_{b}}\right) \nabla \boldsymbol{u}, \nabla \boldsymbol{v}_{m}\right)+\left((\boldsymbol{u} \cdot \nabla) \boldsymbol{u}, \boldsymbol{v}_{m}\right)=\left\langle\boldsymbol{f}, \boldsymbol{v}_{m}\right\rangle_{\Omega}, \\
\forall S_{m} \in \mathbb{W}_{m}, \quad\left(\partial_{t} T^{*}, S_{m}\right)+\alpha\left(\nabla T^{*}, \nabla S_{m}\right)+\left((\boldsymbol{u} \cdot \nabla) T^{*}, S_{m}\right) \\
\quad=\left\langle g, S_{m}\right\rangle_{\Omega}-\left(\partial_{t} \overline{T_{b}}, S_{m}\right)-\alpha\left(\nabla \overline{T_{b}}, \nabla S_{m}\right)-\left((\boldsymbol{u} . \nabla) \overline{T_{b}}, S_{m}\right),
\end{array}
$$

passing to the limit on $m$ is now easy and by density of $\underset{m \in \mathbb{N}}{\cup} \mathbb{V}_{m} \times \mathbb{W}_{m}$ in $V(\Omega) \times H_{0}^{1}(\Omega)$, this yields that the couple $\left(\boldsymbol{u}, T=T^{*}+\overline{T_{b}}\right)$ satisfies the second and third equation of (2.7), which gives (2.6) and $(2.5)$.

2) By integrating the first equation of (2.12) between 0 and $t$, apply (2.10), and define the functional for all $\boldsymbol{v} \in H_{0}^{1}(\Omega)^{d}$ :

$$
\begin{gathered}
L_{t}(\boldsymbol{v})=\int_{0}^{t}\left((\boldsymbol{f}(., s), \boldsymbol{v})-\left(\nu\left(\left(T^{*}+\overline{T_{b}}\right)(., s)\right) \nabla \boldsymbol{u}(., s), \nabla \boldsymbol{v}\right)-((\boldsymbol{u}(., s) . \nabla) \boldsymbol{u}(., s), \boldsymbol{v})\right) d s \\
-(\boldsymbol{u}(., t), \boldsymbol{v})+\left(\boldsymbol{u}_{0}, \boldsymbol{v}\right) .
\end{gathered}
$$


For all $t \in[0, \widetilde{T}]$, this is a continuous linear functional on $H^{1}(\Omega)^{d}$ and, according to $(2.10)-(2.12)$, it vanishes on $V(\Omega)$. Hence, see [11, Chap. I, Lemme 2.1], for each $t \in[0, \widetilde{T}]$, there exists a function $P(t)$ in $L_{\circ}^{2}(\Omega)$ such that:

$$
\begin{gathered}
\forall \boldsymbol{v} \in H_{0}^{1}(\Omega)^{d}, \quad L_{t}(\boldsymbol{v})=-(\operatorname{div} \boldsymbol{v}, P(t)), \\
\|P(t)\|_{L^{2}(\Omega)} \leqslant \sup _{\boldsymbol{v} \in H_{0}^{1}(\Omega)^{d}} \frac{L_{t}(\boldsymbol{v})}{\|\boldsymbol{v}\|_{H^{1}(\Omega)^{d}}} .
\end{gathered}
$$

Now, differentiating (2.18) with respect to $t$, and setting

$$
p=\partial_{t} P(t),
$$

we obtain the first equation of (2.7) with $T=T^{*}+\overline{T_{b}}$. Thus, the variational problems $(2.5)-(2.6)-(2.7)$ and $(2.10)-(2.12)$ are equivalent and have a solution. It is interesting to read in [13, Chap. III, §1.5] analoguous results for the Stokes problem.

Corollary 2.4 If all the assumptions of Theorem 2.3 hold, the solution exhibited in this theorem satisfies,

$$
\begin{array}{r}
\|\boldsymbol{u}\|_{L^{2}\left(0, \widetilde{T} ; H^{1}(\Omega)^{d}\right)}+\|T\|_{L^{2}\left(0, \widetilde{T} ; H^{1}(\Omega)\right)} \leqslant c\left(\left\|T_{0}\right\|_{L^{2}(\Omega)}+\left\|T_{b}^{0}\right\|_{H^{\frac{1}{2}}(\partial \Omega)}+\left\|\boldsymbol{u}_{0}\right\|_{L^{2}(\Omega)^{d}}^{2}+\|\boldsymbol{f}\|_{L^{2}\left(0, \widetilde{T} ; H^{-1}(\Omega)^{d}\right)}^{2}\right. \\
\left.+\left\|T_{b}\right\|_{L^{2}\left(0, \widetilde{T} ; H^{\frac{1}{2}}(\partial \Omega)\right)}^{2}+\|g\|_{L^{2}\left(0, \widetilde{T} ; H^{-1}(\Omega)\right)}\right) .
\end{array}
$$

Proof: The estimate on $\boldsymbol{u}$ being proved in Lemma 2.2, we only consider the estimate on $T$. We take $S$ equal to $T^{*}$ in $(2.12)$, this gives

$$
\left(\partial_{t} T^{*}, T^{*}\right)+\alpha\left(\nabla T^{*}, \nabla T^{*}\right)=\left\langle g, T^{*}\right\rangle_{\Omega}-\left(\partial_{t} \overline{T_{b}}, T^{*}\right)-\alpha\left(\nabla \overline{T_{b}}, \nabla T^{*}\right)-\left((\boldsymbol{u} . \nabla) \overline{T_{b}}, T^{*}\right) .
$$

It follows from the imbedding of $H^{1}(\Omega)$ into $L^{4}(\Omega)$ and the Cauchy-Schwarz inequality, that

$$
\begin{aligned}
\frac{1}{2} \frac{d}{d t}\left\|T^{*}\right\|_{L^{2}(\Omega)}^{2}+\alpha c\left\|T^{*}\right\|_{H^{1}(\Omega)}^{2} & \leqslant\|g\|_{H^{-1}(\Omega)}\left\|T^{*}\right\|_{H^{1}(\Omega)}+\left\|\partial_{t} \overline{T_{b}}\right\|_{L^{2}(\Omega)}\left\|T^{*}\right\|_{L^{2}(\Omega)} \\
& +\alpha c\left\|\overline{T_{b}}\right\|_{H^{1}(\Omega)}\left\|T^{*}\right\|_{H^{1}(\Omega)}+c\|\boldsymbol{u}\|_{H^{1}(\Omega)}\left\|\overline{T_{b}}\right\|_{H^{1}(\Omega)}\left\|T^{*}\right\|_{H^{1}(\Omega)}
\end{aligned}
$$

thus,

$$
\frac{1}{2} \frac{d}{d t}\left\|T^{*}\right\|_{L^{2}(\Omega)}^{2}+\alpha c\left\|T^{*}\right\|_{H^{1}(\Omega)}^{2} \leqslant c\left(\|g\|_{H^{-1}(\Omega)}^{2}+\left\|\partial_{t} \overline{T_{b}}\right\|_{L^{2}(\Omega)}^{2}+\left\|\overline{T_{b}}\right\|_{H^{1}(\Omega)}^{2}+\frac{c^{\prime}}{2}\left(\|\boldsymbol{u}\|_{H^{1}(\Omega)^{d}}^{2}+\left\|\overline{T_{b}}\right\|_{H^{1}(\Omega)}^{2}\right)^{2}\right)
$$

Integrating between 0 and $t$, and by using (2.9) yields,

$$
\begin{aligned}
& \left\|T^{*}(., t)\right\|_{L^{2}(\Omega)}^{2}+\alpha c \int_{0}^{t}\left\|T^{*}(., s)\right\|_{H^{1}(\Omega)}^{2} d s \\
& \leqslant c\left(\left\|T^{*}(., 0)\right\|_{L^{2}(\Omega)}^{2}+\int_{0}^{t}\|g(., s)\|_{H^{-1}(\Omega)}^{2} d s+\int_{0}^{t}\left\|\partial_{t} \overline{T_{b}}(., s)\right\|_{L^{2}(\Omega)}^{2} d s+\int_{0}^{t}\left\|T_{b}(., s)\right\|_{H^{\frac{1}{2}}(\partial \Omega)}^{2} d s\right. \\
& \left.\quad+\frac{c^{\prime}}{2}\left(\int_{0}^{t}\|\boldsymbol{u}(., s)\|_{H^{1}(\Omega) d}^{2} d s+\int_{0}^{t}\left\|T_{b}(., s)\right\|_{H^{\frac{1}{2}}(\partial \Omega)}^{2} d s\right)^{2}\right) .
\end{aligned}
$$

Owing to (2.14) and (2.10),

$$
\begin{array}{r}
\left\|T^{*}\right\|_{L^{2}\left(0, t ; H^{1}(\Omega)\right)} \leqslant c\left(\left\|T_{0}\right\|_{L^{2}(\Omega)}+\left\|T_{b}^{0}\right\|_{H^{\frac{1}{2}}(\partial \Omega)}+\left\|\boldsymbol{u}_{0}\right\|_{L^{2}(\Omega)^{d}}^{2}+\|\boldsymbol{f}\|_{L^{2}\left(0, t ; H^{-1}(\Omega)^{d}\right)}^{2}\right. \\
\left.+\left\|T_{b}\right\|_{H^{1}\left(0, t ; H^{\frac{1}{2}}(\partial \Omega)\right)}^{2}+\|g\|_{L^{2}\left(0, t ; H^{-1}(\Omega)\right)}\right) .
\end{array}
$$


Thus, the desired estimate follows from the triangle inequality.

Since the uniqueness for Navier-Stokes equations remains an open problem in dimension $d=3$, we only consider the case of dimension $d=2$ in the following theorem.

Theorem 2.5 Assume that the function $\nu$ is Lipschitz-continuous, with Lipschitz constant $\nu$. There exist two positive constants $c_{\sharp}^{\Downarrow}$ and $c_{b}^{\star}$ such that

(i) if the data $\left(\boldsymbol{f}, g, T_{b}\right)$ in $L^{2}\left(0, \widetilde{T} ; H^{-1}(\Omega)^{2}\right) \times L^{2}\left(0, \widetilde{T} ; H^{-1}(\Omega)\right) \times L^{2}\left(0, \widetilde{T} ; H^{\frac{1}{2}}(\partial \Omega)\right), \boldsymbol{u}_{0}$ in $L^{2}(\Omega)^{2}$, $T_{0}$ in $L^{2}(\Omega)$ and $T_{b}^{0}$ in $H^{\frac{1}{2}}(\partial \Omega)$ satisfy

$$
\begin{aligned}
c_{\sharp}\left(\left\|T_{0}\right\|_{L^{2}(\Omega)}+\left\|T_{b}^{0}\right\|_{H^{\frac{1}{2}}(\partial \Omega)}+\|\right. & \boldsymbol{u}_{0}\left\|_{L^{2}(\Omega)^{2}}^{2}+\right\| \boldsymbol{f} \|_{L^{2}\left(0, \widetilde{T} ; H^{-1}(\Omega)^{2}\right)}^{2} \\
& \left.+\left\|T_{b}\right\|_{L^{2}\left(0, \widetilde{T} ; H^{\frac{1}{2}}(\partial \Omega)\right)}^{2}+\|g\|_{L^{2}\left(0, \widetilde{T} ; H^{-1}(\Omega)\right)}\right)<1,
\end{aligned}
$$

(ii) if problem $(2.5)-(2.6)-(2.7)$ admits a solution $(\boldsymbol{u}, p, T)$ such that $\boldsymbol{u}$ belongs to $L^{2}\left(0, \widetilde{T} ; W^{1, q}(\Omega)^{2}\right)$ with $q>2$, and satisfies

$$
c_{b} \nu^{\bullet}|\boldsymbol{u}|_{L^{2}\left(0, \widetilde{T} ; W^{1, q}(\Omega)^{2}\right)}<1,
$$

then in dimension $d=2$ this solution is unique.

Proof: We set,

$c_{1}^{\bullet}=c\left(\left\|T_{0}\right\|_{L^{2}(\Omega)}+\left\|T_{b}^{0}\right\|_{H^{\frac{1}{2}(\partial \Omega)}}+\left\|\boldsymbol{u}_{0}\right\|_{L^{2}(\Omega)^{2}}^{2}+\|\boldsymbol{f}\|_{L^{2}\left(0, \widetilde{T} ; H^{-1}(\Omega)^{2}\right)}^{2}+\left\|T_{b}\right\|_{L^{2}\left(0, \widetilde{T} ; H^{\frac{1}{2}}(\partial \Omega)\right)}^{2}+\|g\|_{L^{2}\left(0, \widetilde{T} ; H^{-1}(\Omega)\right)}\right)$.

where $c$ is the constant in (2.20).

Let $\left(\boldsymbol{u}_{1}, p_{1}, T_{1}\right)$ and $\left(\boldsymbol{u}_{2}, p_{2}, T_{2}\right)$ be two solutions of problem $(2.5)-(2.6)-(2.7)$ with $\boldsymbol{u}_{1}$ in $L^{2}\left(0, \widetilde{T} ; W^{1, q}(\Omega)^{2}\right)$ satisfying (2.21).

Setting for a while

$$
\boldsymbol{u}=\boldsymbol{u}_{1}-\boldsymbol{u}_{2}, \quad p=p_{1}-p_{2} \quad \text { and } \quad T=T_{1}-T_{2},
$$

we proceed in three steps.

1) It follows from the third equation in (2.7) that, since $T$ belongs to $H_{0}^{1}(\Omega)$,

$$
\begin{aligned}
\frac{1}{2} \frac{d}{d t}\|T\|_{L^{2}(\Omega)}^{2}+\alpha \int_{\Omega}(\operatorname{grad} T)^{2}(\boldsymbol{x}, t) \mathrm{d} \boldsymbol{x} & =-\int_{\Omega}\left(\left(\boldsymbol{u}_{1} \cdot \nabla\right) T_{1}-\left(\boldsymbol{u}_{2} \cdot \nabla\right) T_{2}\right)(\boldsymbol{x}, t) T(\boldsymbol{x}, t) \mathrm{d} \boldsymbol{x}, \\
& =-\int_{\Omega}\left((\boldsymbol{u} \cdot \nabla) T_{1}\right)(\boldsymbol{x}, t) T(\boldsymbol{x}, t) \mathrm{d} \boldsymbol{x},
\end{aligned}
$$

whence,

$$
\frac{1}{2} \frac{d}{d t}\|T\|_{L^{2}(\Omega)}^{2}+\alpha|T(., t)|_{H^{1}(\Omega)}^{2} \leqslant c_{1}^{\Downarrow} c_{2}^{\Downarrow}|\boldsymbol{u}(., t)|_{H^{1}(\Omega)^{2}}|T(., t)|_{H^{1}(\Omega)},
$$

where $c_{2}^{\star}$ is the square of the norm of the imbedding of $H_{0}^{1}(\Omega)$ into $L^{4}(\Omega)$.

By integrating between 0 and $t$,

$$
\alpha|T|_{L^{2}\left(0, t ; H^{1}(\Omega)\right)} \leqslant c_{1}^{\Downarrow} c_{2}^{\Downarrow}|\boldsymbol{u}|_{L^{2}\left(0, t ; H^{1}(\Omega)^{2}\right)},
$$

2) Similarly, we derive from the first equation in (2.7) that

$$
\begin{array}{r}
\int_{\Omega} \partial_{t} \boldsymbol{u}(\boldsymbol{x}, t) \boldsymbol{u}(\boldsymbol{x}, t) \mathrm{d} \boldsymbol{x}+\int_{\Omega} \nu\left(T_{2}\right)(\boldsymbol{x}, t)(\operatorname{grad} \boldsymbol{u})^{2}(\boldsymbol{x}, t) \mathrm{d} \boldsymbol{x} \\
=-\int_{\Omega}\left(\nu\left(T_{1}\right)-\nu\left(T_{2}\right)\right)(\boldsymbol{x}, t)\left(\operatorname{grad} \boldsymbol{u}_{1}\right)(\boldsymbol{x}, t):(\operatorname{grad} \boldsymbol{u})(\boldsymbol{x}, t) \mathrm{d} \boldsymbol{x} \\
-\int_{\Omega}\left((\boldsymbol{u} \cdot \nabla) \boldsymbol{u}_{1}\right)(\boldsymbol{x}, t) \cdot \boldsymbol{u}(\boldsymbol{x}, t) \mathrm{d} \boldsymbol{x}
\end{array}
$$


Using appropriate Hölder's inequalities and the Lipschitz-continuity of $\nu$ yields

$$
\begin{aligned}
\frac{1}{2} \frac{d}{d t}\|\boldsymbol{u}\|_{L^{2}(\Omega)^{2}}^{2}+ & \nu_{1}|\boldsymbol{u}(., t)|_{H^{1}(\Omega)^{2}}^{2} \\
& \leqslant c_{1}^{\bullet} c_{2}^{\bullet}|\boldsymbol{u}(., t)|_{H^{1}(\Omega)^{2}}^{2}+\nu^{\bullet} c_{3}^{\bullet}\left|\boldsymbol{u}_{1}(., t)\right|_{W^{1, q}(\Omega)^{2}}|T(., t)|_{H^{1}(\Omega)}|\boldsymbol{u}(., t)|_{H^{1}(\Omega)^{2}}
\end{aligned}
$$

where $c_{3}^{\star}$ stands for the norm of the imbedding of $H_{0}^{1}(\Omega)$ into $L^{q^{*}}(\Omega)$, with $\frac{1}{q}+\frac{1}{q^{*}}=\frac{1}{2}$.

By integrating between 0 and $t$ and by using (2.22),

$$
|\boldsymbol{u}|_{L^{2}\left(0, t ; H^{1}(\Omega)^{2}\right)} \leqslant c_{1}^{\Downarrow} c_{2}^{\star} \nu_{1}^{-1}\left(1+\nu^{\bullet} c_{3}^{\star} \alpha^{-1}\left|\boldsymbol{u}_{1}\right|_{L^{2}\left(0, t ; W^{1, q}(\Omega)^{2}\right)}\right)|\boldsymbol{u}|_{L^{2}\left(0, t ; H^{1}(\Omega)^{2}\right)},
$$

By choosing

$$
c_{\sharp}^{\star}=c_{2}^{\star} \nu_{1}^{-1} \quad \text { and } \quad c_{b}^{\star}=c_{1}^{\Downarrow} c_{2}^{\star} \nu_{1}^{-1} c_{3}^{\star} \alpha^{-1},
$$

such that

$$
c_{1}^{\bullet} c_{2}^{\bullet} \nu_{1}^{-1}\left(1+\nu \bullet c_{3}^{\star} \alpha^{-1}\left|\boldsymbol{u}_{1}\right|_{L^{2}\left(0, t ; W^{1, q}(\Omega)^{2}\right)}\right)<1,
$$

we obtain that $\boldsymbol{u}$ is zero, so that $\boldsymbol{u}_{1}$ and $\boldsymbol{u}_{2}$ are equal.

3) It then follows from (2.22) that $T_{1}$ and $T_{2}$ are equal. Finally, the function $p$ satisfies

$$
\forall \boldsymbol{v} \in H_{0}^{1}(\Omega)^{2}, \quad-\int_{\Omega}(\operatorname{div} \boldsymbol{v})(\boldsymbol{x}) p(\boldsymbol{x}) \mathrm{d} \boldsymbol{x}=0,
$$

so that it is zero (see [11, Chap. I, §2] for instance). Thus $p_{1}$ and $p_{2}$ coincide.

This concludes the proof.

\section{The discrete problem}

As already explained we propose a discretization of problem (2.5)-(2.6)-(2.7). For its a priori analysis, it is simpler to work directly on the fully discrete problem. However we prefer to split the discretization into two steps: First a semi-discretization in time, and next the full discretization.

\subsection{The time semi-discrete problem.}

Since we intend to work with non uniform time steps, we introduce a partition of the interval $[0, \widetilde{T}]$ into subintervals $\left[t_{j-1}, t_{j}\right], 1 \leqslant j \leqslant J$, such that

$$
0=t_{0}<t_{1}<\ldots<t_{J}=\widetilde{T} .
$$

We denote by $\tau_{j}$ the time step $t_{j}-t_{j-1}$, by $\tau$ the $J$-tuple $\left(\tau_{1}, \ldots, \tau_{J}\right)$ and by $|\tau|$ the maximum of the $\tau_{j}, 1 \leqslant j \leqslant J$.

As explained in the introduction, the time discretization of the problem relies on the use of a backward Euler's sheme, where the nonlinear terms $(\boldsymbol{u} . \nabla) \boldsymbol{u}$ and $(\boldsymbol{u} . \nabla) T$ are treated in an implicit way and for the sake of simplicity, we shall present our analysis by approximating the nonlinear term by $\nu\left(T^{j-1}\right)$. Thus, for any data $\left(\boldsymbol{f}, g, T_{b}\right)$ in $\mathscr{C}^{0}\left(0, \widetilde{T} ; H^{-1}(\Omega)^{d}\right) \times \mathscr{C}^{0}\left(0, \widetilde{T} ; H^{-1}(\Omega)\right) \times \mathscr{C}^{0}\left(0, \widetilde{T} ; H^{\frac{1}{2}}(\partial \Omega)\right)$, $T_{0}$ in $H^{1}(\Omega)$ and $\boldsymbol{u}_{0}$ in $H_{0}^{1}(\Omega)^{d}$ satisfying (2.4), we consider the following scheme:

Find $\left(\boldsymbol{u}^{j}\right)_{0 \leqslant j \leqslant J}$ in $\left(H_{0}^{1}(\Omega)^{d}\right)^{J+1},\left(p^{j}\right)_{1 \leqslant j \leqslant J}$ in $\left(L_{\circ}^{2}(\Omega)\right)^{J}$ and $\left(T^{j}\right)_{0 \leqslant j \leqslant J}$ in $\left(H^{1}(\Omega)\right)^{J+1}$ such that

$$
\boldsymbol{u}^{0}=\boldsymbol{u}_{0} \quad \text { and } \quad T^{0}=T_{0} \quad \text { in } \Omega,
$$

such that, for all $j, 1 \leqslant j \leqslant J$

$$
T^{j}=T_{b}^{j} \quad \text { on } \partial \Omega,
$$


and, for all $j, 1 \leqslant j \leqslant J$

$$
\begin{aligned}
& \forall \boldsymbol{v} \in H_{0}^{1}(\Omega)^{d}, \quad\left(\boldsymbol{u}^{j}, \boldsymbol{v}\right)+\tau_{j}\left(\nu\left(T^{j-1}\right) \nabla \boldsymbol{u}^{j}, \nabla \boldsymbol{v}\right)+\tau_{j}\left(\left(\boldsymbol{u}^{j} . \nabla\right) \boldsymbol{u}^{j}, \boldsymbol{v}\right) \\
& \forall q \in \tau_{j}\left(\operatorname{div} \boldsymbol{v}, p^{j}\right)=\left(\boldsymbol{u}^{j-1}, \boldsymbol{v}\right)+\tau_{j}\left\langle\boldsymbol{f}^{j}, \boldsymbol{v}\right\rangle_{\Omega}, \\
& \forall S \in H_{0}^{1}(\Omega), \quad\left(T^{j}, S\right)+\alpha \tau_{j}\left(\nabla T^{j}, \nabla S\right)+\tau_{j}\left(\left(\boldsymbol{u}^{j}, \nabla\right) T^{j}, S\right)=\left(T^{j-1}, S\right)+\tau_{j}\left\langle g^{j}, S\right\rangle_{\Omega},
\end{aligned}
$$

where $\boldsymbol{f}^{j}=\boldsymbol{f}\left(., t_{j}\right), g^{j}=g\left(., t_{j}\right)$ and $T_{b}^{j}=T_{b}\left(., t_{j}\right)$.

As for the exact problem, it is suitable to lift the boundary data $T_{b}^{j}$ : For $0 \leqslant j \leqslant J$, we $\operatorname{set} \bar{T}_{b}^{j}=\mathcal{R} T_{b}{ }^{j}$, where the operator $\mathcal{R}$ is intoduced in Section 2, and we have

$$
\left\|{\overline{T_{b}}}^{j}\right\|_{H^{1}(\Omega)} \leqslant c_{\diamond}\left\|T_{b}^{j}\right\|_{H^{\frac{1}{2}(\partial \Omega)}},
$$

with the same constant $c$ as in (2.9). Thus, when setting $T^{* j}=T^{j}-{\overline{T_{b}}}^{j}$, if $\left(\boldsymbol{u}^{j}, p^{j}, T^{j}\right)$ is any solution of $(3.1)-(3.2)-(3.3)$, the sequence $\left(\boldsymbol{u}^{j}, T^{* j}\right)$ belongs to $V(\Omega)^{J+1} \times\left(H_{0}^{1}(\Omega)^{d}\right)^{J+1}$ and satisfies

$$
\boldsymbol{u}^{0}=\boldsymbol{u}_{0} \quad \text { and } \quad T^{* 0}=T^{0}-{\overline{T_{b}}}^{0} \quad \text { in } \Omega,
$$

$$
\begin{array}{r}
\forall \boldsymbol{v} \in V(\Omega), \quad\left(\boldsymbol{u}^{j}, \boldsymbol{v}\right)+\tau_{j}\left(\nu\left(T^{* j-1}+{\overline{T_{b}}}^{j-1}\right) \nabla \boldsymbol{u}^{j}, \nabla \boldsymbol{v}\right)+\tau_{j}\left(\left(\boldsymbol{u}^{j} . \nabla\right) \boldsymbol{u}^{j}, \boldsymbol{v}\right)=\left(\boldsymbol{u}^{j-1}, \boldsymbol{v}\right)+\tau_{j}\left\langle\boldsymbol{f}^{j}, \boldsymbol{v}\right\rangle_{\Omega}, \\
\forall S \in H_{0}^{1}(\Omega), \quad\left(T^{* j}, S\right)+\alpha \tau_{j}\left(\nabla T^{* j}, \nabla S\right)+\tau_{j}\left(\left(\boldsymbol{u}^{j} . \nabla\right) T^{* j}, S\right)=\left(T^{* j-1}, S\right)+\tau_{j}\left\langle g^{j}, S\right\rangle_{\Omega}-\left({\overline{T_{b}}}^{j}, S\right) \\
+\left({\overline{T_{b}}}^{j-1}, S\right)-\alpha \tau_{j}\left(\nabla{\overline{T_{b}}}^{j}, \nabla S\right)-\tau_{j}\left(\left(\boldsymbol{u}^{j} . \nabla\right){\overline{T_{b}}}^{j}, S\right) .
\end{array}
$$

We skip the proof of existence of $\left(\boldsymbol{u}^{j}, T^{* j}\right)$ which is rather standard (and simpler than in Section 2). Moreover, if $\left(\boldsymbol{u}^{j}, T^{* j}\right)$ is any solution of (3.5)-(3.6), we define the linear mapping

$$
L_{j}(\boldsymbol{v})=\left\langle\boldsymbol{f}^{j}, \boldsymbol{v}\right\rangle_{\Omega}-\left(\nu\left(T^{* j-1}+\bar{T}_{b}^{j-1}\right) \nabla \boldsymbol{u}^{j}, \nabla \boldsymbol{v}\right)-\left(\left(\boldsymbol{u}^{j} . \nabla\right) \boldsymbol{u}^{j}, \boldsymbol{v}\right)-\frac{1}{\tau_{j}}\left(\boldsymbol{u}^{j}-\boldsymbol{u}^{j-1}, \boldsymbol{v}\right) .
$$

Clearly, the mapping $\boldsymbol{v} \mapsto L_{j}(\boldsymbol{v})$ is a continuous linear fonctional on $H_{0}^{1}(\Omega)^{d}$ that vanishes on $V(\Omega)$. The inf-sup condition (2.8) implies that there exists an element $p^{j}$ in $L_{\circ}^{2}(\Omega)$ such that

$$
\begin{array}{r}
\forall \boldsymbol{v} \in H_{0}^{1}(\Omega)^{d}, \quad L_{j}(\boldsymbol{v})=-\left(\operatorname{div} \boldsymbol{v}, p^{j}\right), \\
\left\|p^{j}\right\|_{L^{2}(\Omega)} \leqslant \sup _{v \in H_{0}^{1}(\Omega)^{d}} \frac{L_{j}(\boldsymbol{v})}{\|\boldsymbol{v}\|_{H^{1}(\Omega)^{d}}} .
\end{array}
$$

Hence $\left(\boldsymbol{u}^{j}, p^{j}, T^{j}=T^{* j}+{\overline{T_{b}}}^{j}\right)$ is a solution of $(3.1)-(3.2)-(3.3)$. This enables us to state the next proposition.

Proposition 3.1 Assume that the data $\left(\boldsymbol{f}, g, T_{b}\right)$ belong to

$$
\mathscr{C}^{0}\left(0, \widetilde{T}, H^{-1}(\Omega)^{d}\right) \times \mathscr{C}^{0}\left(0, \widetilde{T}, H^{-1}(\Omega)\right) \times \mathscr{C}^{0}\left(0, \widetilde{T} ; H^{\frac{1}{2}}(\partial \Omega)\right),
$$

that the initial velocity $\boldsymbol{u}_{0}$ belongs to $L^{2}(\Omega)^{d}$ and the initial temperature $T_{0}$ belongs to $H^{1}(\Omega)$. Then, problem (3.1)-(3.2)-(3.3) has a solution $\left(\boldsymbol{u}^{j}, p^{j}, T^{j}\right)$ such that

$$
\boldsymbol{u}^{j} \in H_{0}^{1}(\Omega)^{d}, 1 \leqslant j \leqslant J, \quad p^{j} \in L_{\circ}^{2}(\Omega), 1 \leqslant j \leqslant J, \quad \text { and } \quad T^{j} \in H^{1}(\Omega), 0 \leqslant j \leqslant J .
$$

Moreover the sequence of velocities $\left(\boldsymbol{u}^{j}\right)_{1 \leqslant j \leqslant J}$ satisfies for $j \geqslant 1$

$$
\left\|\boldsymbol{u}^{j}\right\|_{H^{1}(\Omega)^{d}} \leqslant\left\|\boldsymbol{u}^{0}\right\|_{L^{2}(\Omega)^{d}}+\sqrt{\frac{1}{c \nu_{1}}}\left(\sum_{m=1}^{j} \tau_{m}\left\|\boldsymbol{f}^{m}\right\|_{H^{-1}(\Omega)^{d}}^{2}\right)^{\frac{1}{2}} .
$$


Proof: We only prove the bound (3.9). We take $\boldsymbol{v}=\boldsymbol{u}^{j}$ in the first equation of (3.3) and we use the relation

$$
a(a-b)=\frac{1}{2} a^{2}+\frac{1}{2}(a-b)^{2}-\frac{1}{2} b^{2},
$$

we obtain

$$
\begin{array}{r}
\frac{1}{2}\left(\left\|\boldsymbol{u}^{j}\right\|_{L^{2}(\Omega)^{d}}^{2}-\left\|\boldsymbol{u}^{j-1}\right\|_{L^{2}(\Omega)^{d}}^{2}+\left\|\boldsymbol{u}^{j}-\boldsymbol{u}^{j-1}\right\|_{L^{2}(\Omega)^{d}}^{2}\right)+c \nu_{1} \tau_{j}\left\|\boldsymbol{u}^{j}\right\|_{H^{1}(\Omega)^{d}}^{2} \\
\leqslant \tau_{j}\left\|\boldsymbol{f}^{j}\right\|_{H^{-1}(\Omega)^{d}}\left\|\boldsymbol{u}^{j}\right\|_{H^{1}(\Omega)^{d}}
\end{array}
$$

whence

$$
\left\|\boldsymbol{u}^{j}\right\|_{L^{2}(\Omega)^{d}}^{2}-\left\|\boldsymbol{u}^{j-1}\right\|_{L^{2}(\Omega)^{d}}^{2}+\left\|\boldsymbol{u}^{j}-\boldsymbol{u}^{j-1}\right\|_{L^{2}(\Omega)^{d}}^{2}+c \nu_{1} \tau_{j}\left\|\boldsymbol{u}^{j}\right\|_{H^{1}(\Omega)^{d}}^{2} \leqslant \frac{\tau_{j}}{c \nu_{1}}\left\|\boldsymbol{f}^{j}\right\|_{H^{-1}(\Omega)^{d}}^{2} .
$$

Summing this inequality over $j$, we obtain

$$
\begin{aligned}
\left\|\boldsymbol{u}^{j}\right\|_{L^{2}(\Omega)^{d}}^{2}+\sum_{m=1}^{j}\left\|\boldsymbol{u}^{m}-\boldsymbol{u}^{m-1}\right\|_{L^{2}(\Omega)^{d}}^{2}+c \nu_{1} \sum_{m=1}^{j} & \tau_{m}\left\|\boldsymbol{u}^{m}\right\|_{H^{1}(\Omega)^{d}}^{2} \\
& \leqslant\left\|\boldsymbol{u}^{0}\right\|_{L^{2}(\Omega)^{d}}^{2}+\frac{1}{c \nu_{1}} \sum_{m=1}^{j} \tau_{m}\left\|\boldsymbol{f}^{m}\right\|_{H^{-1}(\Omega)^{d}}^{2} .
\end{aligned}
$$

This concludes the proof.

\subsection{The time and space discrete problem}

We are now interested in the discretization of problem $(3.1)-(3.2)-(3.3)$ in the case where $\Omega=$ ]$-1,1\left[d, d=2\right.$ or 3 . Let $N$ be an integer $\geqslant 2$, we introduce the space $\mathbb{P}_{N}(\Omega)$ of polynomials with $d$ variables and degree $\leqslant N$ with respect to each variable and the space $\mathbb{P}_{N}^{0}(\Omega)$ of polynomials in $\mathbb{P}_{N}(\Omega)$ vanishing on the boundary of $\Omega$. Relying on theses definitions, we introduce the discrete spaces

$$
\begin{aligned}
\mathbb{X}_{N}=\mathbb{P}_{N}^{0}(\Omega)^{d}, \mathbb{M}_{N}=\mathbb{P}_{N-2}(\Omega) & \cap L_{\circ}^{2}(\Omega), \\
& \mathbb{Y}_{N}=\mathbb{P}_{N}(\Omega), \quad \mathbb{Y}_{N}^{0}=\mathbb{Y}_{N} \cap H_{0}^{1}(\Omega) .
\end{aligned}
$$

The reason for the choice of the space $\mathbb{M}_{N}$ is that it does not contain spurious modes, see [5, Chap V]. We introduce the space $\mathbb{P}_{N}(-1,1)$ of restrictions to $[-1,1]$ of polynomials with degree $\leqslant N$. Setting $\xi_{0}=-1$ and $\xi_{N}=1$, we consider the $N-1$ nodes $\xi_{m}, 1 \leqslant m \leqslant N-1$, and the $N+1$ weights $\rho_{m}$, $0 \leqslant m \leqslant N$, of the Gauss-Lobatto quadrature formula. We recall that the following equality holds

$$
\forall \phi \in \mathbb{P}_{2 N-1}(-1,1), \quad \int_{-1}^{1} \phi(\zeta) \mathrm{d} \zeta=\sum_{i=0}^{N} \phi\left(\xi_{i}\right) \rho_{i} .
$$

We also recall $[6$, Chap IV, Cor. 1.10] the following property, which is useful in what follows

$$
\forall \phi_{N} \in \mathbb{P}_{N}(-1,1), \quad\left\|\phi_{N}\right\|_{L^{2}(-1,1)}^{2} \leqslant \sum_{i=0}^{N} \phi_{N}^{2}\left(\xi_{i}\right) \rho_{i} \leqslant 3\left\|\phi_{N}\right\|_{L^{2}(-1,1)}^{2} .
$$

Relying on this formula, we introduce the discrete product, defined on continuous functions $u$ and $v$ by

$$
(u, v)_{N}= \begin{cases}\sum_{i=0}^{N} \sum_{j=0}^{N} u\left(\xi_{i}, \xi_{j}\right) v\left(\xi_{i}, \xi_{j}\right) \rho_{i} \rho_{j} & \text { if } d=2, \\ \sum_{i=0}^{N} \sum_{j=0}^{N} \sum_{k=0}^{N} u\left(\xi_{i}, \xi_{j}, \xi_{k}\right) v\left(\xi_{i}, \xi_{j}, \xi_{k}\right) \rho_{i} \rho_{j} \rho_{k} & \text { if } d=3 .\end{cases}
$$


It follows from (3.12) that it is a scalar product on $\mathbb{P}_{N}(\Omega)$. Let $\mathcal{I}_{N}$ denote the Lagrange interpolation operator at the nodes of the grid

$$
\Sigma_{N}=\left\{\begin{array}{lr}
\left\{\left(\xi_{i}, \xi_{j}\right) ; \quad 0 \leqslant i, j \leqslant N\right\} & \text { if } d=2 \\
\left\{\left(\xi_{i}, \xi_{j}, \xi_{k}\right) ; \quad 0 \leqslant i, j, k \leqslant N\right\} & \text { if } d=3
\end{array}\right.
$$

with values in the space $\mathbb{P}_{N}(\Omega)$. Finally, let $i_{N}^{\partial \Omega}$ stand for the Lagrange interpolation operator at the nodes of $\Sigma_{N} \cap \partial \Omega$ with values in the space of traces of $\mathbb{P}_{N}(\Omega)$.

We now assume that the function $T_{b}$ is continuous on $\partial \Omega \times[0, \widetilde{T}]$ and $\boldsymbol{f}, g$ are continuous on $\bar{\Omega} \times[0, \widetilde{T}]$. Thus the fully discrete problem is constructed from (3.1)-(3.2) - (3.3) by using the Galerkin method combined with numerical integration. It reads

Find $\left(\boldsymbol{u}_{N}^{j}\right)_{0 \leqslant j \leqslant J}$ in $\mathbb{X}_{N}^{J+1},\left(p_{N}^{j}\right)_{1 \leqslant j \leqslant J}$ in $\mathbb{M}_{N}^{J}$ and $\left(T_{N}^{j}\right)_{0 \leqslant j \leqslant J}$ in $\mathbb{Y}_{N}^{J+1}$ such that

$$
\boldsymbol{u}_{N}^{0}=\mathcal{I}_{N} \boldsymbol{u}_{0} \quad \text { and } \quad T_{N}^{0}=\mathcal{I}_{N} T_{0} \quad \text { in } \Omega,
$$

for $1 \leqslant j \leqslant J$,

$$
T_{N}^{j}=i_{N}^{\partial \Omega} T_{b}^{j} \quad \text { on } \partial \Omega
$$

and, for $1 \leqslant j \leqslant J$,

$$
\begin{aligned}
& \forall \boldsymbol{v}_{N} \in \mathbb{X}_{N}, \quad\left(\boldsymbol{u}_{N}^{j}, \boldsymbol{v}_{N}\right)_{N}+\tau_{j}\left(\nu\left(T_{N}^{j-1}\right) \operatorname{grad} \boldsymbol{u}_{N}^{j}, \operatorname{grad} \boldsymbol{v}_{N}\right)_{N}+\tau_{j}\left(\left(\boldsymbol{u}_{N}^{j} \cdot \nabla\right) \boldsymbol{u}_{N}^{j}, \boldsymbol{v}_{N}\right)_{N} \\
& -\tau_{j}\left(\operatorname{div} \boldsymbol{v}_{N}, p_{N}^{j}\right)_{N}=\left(\boldsymbol{u}_{N}^{j-1}, \boldsymbol{v}_{N}\right)_{N}+\tau_{j}\left(\boldsymbol{f}^{j}, \boldsymbol{v}_{N}\right)_{N}, \\
& \forall q_{N} \in \mathbb{M}_{N}, \quad-\left(\operatorname{div} \boldsymbol{u}_{N}^{j}, q_{N}\right)_{N}=0, \\
& \forall S_{N} \in \mathbb{Y}_{N}^{0}, \quad\left(T_{N}^{j}, S_{N}\right)_{N}+\alpha \tau_{j}\left(\operatorname{grad} T_{N}^{j}, \operatorname{grad} S_{N}\right)_{N}+\tau_{j}\left(\left(\boldsymbol{u}_{N}^{j} \cdot \nabla\right) T_{N}^{j}, S_{N}\right)_{N} \\
& =\left(T_{N}^{j-1}, S_{N}\right)_{N}+\tau_{j}\left(g^{j}, S_{N}\right)_{N} \text {. }
\end{aligned}
$$

The existence of a solution can be derived by similar arguments as in Section 2, however we prefer to follow the approach of [7] to obtain directly more precise results.

We recall the existence of a discrete inf-sup condition between the spaces $\mathbb{X}_{N}$ and $\mathbb{M}_{N}$, see $[5$, Chap. V, Thm 25.7]

$$
\forall q_{N} \in \mathbb{M}_{N}, \quad \sup _{\boldsymbol{v}_{N} \in \mathbb{X}_{N}} \frac{\int_{\Omega}\left(\operatorname{div} \boldsymbol{v}_{N}\right)(\boldsymbol{x}) q_{N}(\boldsymbol{x}) \mathrm{d} \boldsymbol{x}}{\left\|\boldsymbol{v}_{N}\right\|_{H^{1}(\Omega)^{d}}} \geqslant c N^{-(d-1) / 2}\left\|q_{N}\right\|_{L^{2}(\Omega)} .
$$

As for the continuous problem, we introduce the kernel

$$
V_{N}(\Omega)=\left\{\boldsymbol{v}_{N} \in \mathbb{X}_{N} ; \forall q_{N} \in \mathbb{M}_{N},\left(\operatorname{div} \boldsymbol{v}_{N}, q_{N}\right)_{N}=0\right\} .
$$

\section{A priori error analysis}

\subsection{New formulation}

For any real-valued measurable fonction $\theta$ on $\Omega$, we introduce the modified Stokes operator $\mathcal{S}(\theta)$, which associates with any data $\boldsymbol{F}$ in $L^{2}\left(0, \widetilde{T} ; H^{-1}(\Omega)^{d}\right)$ and $\boldsymbol{u}_{0}$ in $H_{0}^{1}(\Omega)^{d}$ the part $\boldsymbol{u}$ of the solution $(\boldsymbol{u}, p)$ of the generalized Stokes problem

Find $(\boldsymbol{u}, p)$ in $L^{2}\left(0, \widetilde{T} ; H_{0}^{1}(\Omega)^{d}\right) \times L^{2}\left(0, \widetilde{T} ; L_{\circ}^{2}(\Omega)\right)$ such that

$$
\boldsymbol{u}=\mathbf{0} \quad \text { on } \partial \Omega \times] 0, \widetilde{T}\left[\text { and }\left.\quad \boldsymbol{u}\right|_{t=0}=\boldsymbol{u}_{0} \quad \text { in } \Omega,\right.
$$


and, for a.e. $t$ in $] 0, \widetilde{T}[$,

$$
\begin{aligned}
& \forall \boldsymbol{v} \in H_{0}^{1}(\Omega)^{d}, \quad \int_{\Omega} \partial_{t} \boldsymbol{u}(\boldsymbol{x}, t) \boldsymbol{v}(\boldsymbol{x}) \mathrm{d} \boldsymbol{x}+\int_{\Omega} \nu(\theta) \nabla \boldsymbol{u}(\boldsymbol{x}) \nabla \boldsymbol{v}(\boldsymbol{x}) \mathrm{d} \boldsymbol{x} \\
&-\int_{\Omega}(\operatorname{div} \boldsymbol{v})(\boldsymbol{x}) p(\boldsymbol{x}) \mathrm{d} \boldsymbol{x}=\langle\boldsymbol{F}(., t), \boldsymbol{v}\rangle_{\Omega}, \\
& \forall q \in L_{\circ}^{2}(\Omega), \quad-\int_{\Omega}(\operatorname{div} \boldsymbol{u})(\boldsymbol{x}) q(\boldsymbol{x}) \mathrm{d} \boldsymbol{x}=0 .
\end{aligned}
$$

We also consider the operator $\widetilde{\mathcal{S}}(\theta)$ which associates with any data $\boldsymbol{F}$ in $L^{2}\left(0, \widetilde{T} ; H^{-1}(\Omega)^{d}\right)$ and $\boldsymbol{u}_{0}$ in $H_{0}^{1}(\Omega)^{d}$ the part $p$ of the solution $(\boldsymbol{u}, p)$ of this same problem.

We introduce the inverse $\mathcal{L}$ of the Laplace operator which associates with any data $\left(G, T_{b}, T_{0}\right)$ in $L^{2}\left(0, \widetilde{T} ; H^{-1}(\Omega)\right) \times L^{2}\left(0, \widetilde{T} ; H^{\frac{1}{2}}(\partial \Omega)\right) \times H^{1}(\Omega)$ the solution $T$ of the problem

Find $T$ in $L^{2}\left(0, \widetilde{T} ; H^{1}(\Omega)\right)$ such that

$$
\left.T=T_{b} \quad \text { on } \partial \Omega \times\right] 0, \widetilde{T}\left[\text { and }\left.\quad T\right|_{t=0}=T_{0} \quad \text { in } \Omega, .\right.
$$

and, for a.e. $t$ in $] 0, \widetilde{T}[$,

$$
\forall S \in H_{0}^{1}(\Omega), \quad \int_{\Omega} \partial_{t} T(\boldsymbol{x}, t) S(\boldsymbol{x}) \mathrm{d} \boldsymbol{x}+\alpha \int_{\Omega} \nabla T(\boldsymbol{x}, t) . \nabla S(\boldsymbol{x}) \mathrm{d} \boldsymbol{x}=\langle G(., t), S\rangle_{\Omega}
$$

Thus it is readily checked that, when setting $U=(\boldsymbol{u}, T)$ and with this notation, problem $(2.5)-(2.6)-(2.7)$ can be written equivalently as

$$
\mathcal{F}(U)=U-\left(\begin{array}{cc}
\mathcal{S}(T) & 0 \\
0 & \mathcal{L}
\end{array}\right) G(U)=0
$$

with

$$
G(U)=\left(\begin{array}{c}
\left(\mathcal{G}_{1}(U), \boldsymbol{u}_{0}\right) \\
\left(\mathcal{G}_{2}(U), T_{b}, T_{0}\right)
\end{array}\right) \quad \text { and } \quad\left(\begin{array}{c}
\mathcal{G}_{1}(U) \\
\mathcal{G}_{2}(U)
\end{array}\right)=\left(\begin{array}{l}
\boldsymbol{f}-(\boldsymbol{u} \cdot \nabla) \boldsymbol{u} \\
g-(\boldsymbol{u} \cdot \nabla) T
\end{array}\right)
$$

\subsubsection{About the time discretization}

From now on, we denote by $\boldsymbol{u}_{\tau}$ and $T_{\tau}$ the functions which are affine on each interval $\left[t_{j-1}, t_{j}\right]$ and equal to $\boldsymbol{u}^{j}$ and $T^{j}$ respectively at each time $t_{j}, 0 \leqslant j \leqslant J$, and by $p_{\tau}$ the function which is piecewise constant equal to $p^{j}$ on each interval $\left.] t_{j-1}, t_{j}\right], 1 \leqslant j \leqslant J$. For each function $\boldsymbol{v}$ continuous on $[0, \widetilde{T}]$, we also introduce the functions $\pi_{\tau}^{+} \boldsymbol{v}$ and $\pi_{\tau}^{-} \boldsymbol{v}$ which are constant, equal to $\boldsymbol{v}\left(t_{j}\right)$ and $\boldsymbol{v}\left(t_{j-1}\right)$, respectively, on each interval $\left.] t_{j-1}, t_{j}\right], 1 \leqslant j \leqslant J$.

Let $\mathcal{S}_{\tau}(\theta)$ denote the following semi-discrete operator: For any data $\boldsymbol{F}$ in $\mathscr{C}^{0}\left(0, \widetilde{T} ; H^{-1}(\Omega)^{d}\right)$ and $\boldsymbol{u}_{0}$ in $H_{0}^{1}(\Omega)^{d}, \mathcal{S}_{\tau}(\theta)\left(\boldsymbol{F}, \boldsymbol{u}_{0}\right)$ is equal to the function $\boldsymbol{u}_{\tau}$ associated with the solution of the semi-discrete problem $\left(\boldsymbol{u}^{j}, p^{j}\right)$ solutions of

Find $\left(\boldsymbol{u}^{j}\right)_{0 \leqslant j \leqslant J}$ in $\left(H_{0}^{1}(\Omega)^{d}\right)^{J+1}$ and $\left(p^{j}\right)_{1 \leqslant j \leqslant J}$ in $\left(L_{\circ}^{2}(\Omega)\right)^{J}$ such that

$$
\boldsymbol{u}^{j}=\mathbf{0} \quad \text { on } \partial \Omega, 1 \leqslant j \leqslant J \quad \text { and } \quad \boldsymbol{u}^{0}=\boldsymbol{u}_{0} \quad \text { in } \Omega,
$$


and, for $1 \leqslant j \leqslant J$,

$$
\begin{gathered}
\forall \boldsymbol{v} \in H_{0}^{1}(\Omega)^{d}, \quad \int_{\Omega}\left(\frac{\boldsymbol{u}^{j}-\boldsymbol{u}^{j-1}}{\tau_{j}}\right)(\boldsymbol{x}) \boldsymbol{v}(\boldsymbol{x}) \mathrm{d} \boldsymbol{x}+\int_{\Omega} \nu(\theta) \nabla \boldsymbol{u}^{j}(\boldsymbol{x}) \nabla \boldsymbol{v}(\boldsymbol{x}) \mathrm{d} \boldsymbol{x} \\
-\int_{\Omega}(\operatorname{div} \boldsymbol{v})(\boldsymbol{x}) p^{j}(\boldsymbol{x}) \mathrm{d} \boldsymbol{x}=\left\langle\boldsymbol{F}\left(., t_{j}\right), \boldsymbol{v}\right\rangle_{\Omega}, \\
\forall q \in L_{\circ}^{2}(\Omega), \quad-\int_{\Omega}\left(\operatorname{div} \boldsymbol{u}^{j}\right)(\boldsymbol{x}) q(\boldsymbol{x}) \mathrm{d} \boldsymbol{x}=0 .
\end{gathered}
$$

We also consider the operator $\widetilde{\mathcal{S}_{\tau}}(\theta)$ which associates with any datum $\boldsymbol{F}$ in $\mathscr{C}^{0}\left(0, \widetilde{T} ; H^{-1}(\Omega)^{d}\right)$ and $\boldsymbol{u}_{0}$ in $H_{0}^{1}(\Omega)^{d}$ the part $p_{\tau}$ of the solution $\left(\boldsymbol{u}^{j}, p^{j}\right)$ of this same problem.

Let finally $\mathcal{L}_{\tau}$ denote the semi-discrete operator: For any data $\left(G, T_{b}, T_{0}\right)$ in $\mathscr{C}^{0}\left(0, \widetilde{T} ; H^{-1}(\Omega)\right) \times$ $\mathscr{C}^{0}\left(0, \widetilde{T} ; H^{\frac{1}{2}}(\partial \Omega)\right) \times H^{1}(\Omega), \mathcal{L}_{\tau}\left(G, T_{b}, T_{0}\right)$ is equal to the function $T_{\tau}$ associated with the $T^{j}$ solutions of

Find $\left(T^{j}\right)_{0 \leqslant j \leqslant J}$ in $\left(H^{1}(\Omega)\right)^{J+1}$ such that

$$
T^{j}(., t)=T_{b}\left(., t_{j}\right) \quad \text { on } \partial \Omega, 1 \leqslant j \leqslant J \quad \text { and } \quad T^{0}=T_{0} \quad \text { in } \Omega,
$$

and, for $1 \leqslant j \leqslant J$,

$$
\forall S \in H_{0}^{1}(\Omega), \quad \int_{\Omega}\left(\frac{T^{j}-T^{j-1}}{\tau_{j}}\right)(\boldsymbol{x}) S(\boldsymbol{x}) \mathrm{d} \boldsymbol{x}+\alpha \int_{\Omega} \nabla T^{j}(\boldsymbol{x}) . \nabla S(\boldsymbol{x}) \mathrm{d} \boldsymbol{x}=\left\langle G\left(., t_{j}\right), S\right\rangle_{\Omega}
$$

Thus, when setting $U_{\tau}=\left(\boldsymbol{u}_{\tau}, T_{\tau}\right)$ problem (3.1)-(3.2)-(3.3) can equivalently be written

$$
\mathcal{F}_{\tau}\left(U_{\tau}\right)=U_{\tau}-\left(\begin{array}{cc}
\mathcal{S}_{\tau}\left(\pi_{\tau}^{-} T_{\tau}\right) & 0 \\
0 & \mathcal{L}_{\tau}
\end{array}\right) G\left(U_{\tau}\right)=0
$$

where $G$ is defined in (4.6).

Now we estimate the error on the velocity between the semi-discrete scheme (4.7)-(4.8) and the continuous problem (4.1)-(4.2). The error equation is obtained by subtracting (4.8) from (4.2) at time $t_{j}$. Thus the sequence $\left(\boldsymbol{e}_{\boldsymbol{u}}^{j}\right)_{0 \leqslant j \leqslant J}$, defined by $\boldsymbol{e}_{\boldsymbol{u}}^{j}=\boldsymbol{u}\left(., t_{j}\right)-\boldsymbol{u}^{j}$ satisfies $\boldsymbol{e}_{\boldsymbol{u}}^{0}=\mathbf{0}$ and, for $1 \leqslant j \leqslant J$,

$$
\begin{aligned}
& \forall \boldsymbol{v} \in H_{0}^{1}(\Omega)^{d}, \quad\left(\boldsymbol{e}_{\boldsymbol{u}}^{j}, \boldsymbol{v}\right)+\tau_{j}\left(\nu(\theta) \nabla \boldsymbol{e}_{\boldsymbol{u}}^{j}, \nabla \boldsymbol{v}\right)-\tau_{j} \int_{\Omega}(\operatorname{div} \boldsymbol{v})(\boldsymbol{x})\left(p\left(., t_{j}\right)-p^{j}\right)(\boldsymbol{x}) d \boldsymbol{x} \\
& =\left(\boldsymbol{e}_{\boldsymbol{u}}^{j-1}, \boldsymbol{v}\right)+\tau_{j}\left\langle\boldsymbol{\varepsilon}_{\boldsymbol{u}}^{j}, \boldsymbol{v}\right\rangle_{\Omega}, \\
& \forall q \in L_{\circ}^{2}(\Omega), \quad-\int_{\Omega}\left(\operatorname{div} \boldsymbol{e}_{\boldsymbol{u}}^{j}\right)(\boldsymbol{x}) q(\boldsymbol{x}) d \boldsymbol{x}=0 .
\end{aligned}
$$

where the consistency error $\varepsilon_{u}^{j}$ is given by

$$
\varepsilon_{\boldsymbol{u}}^{j}=\frac{\boldsymbol{u}\left(., t_{j}\right)-\boldsymbol{u}\left(., t_{j-1}\right)}{\tau_{j}}-\left(\partial_{t} \boldsymbol{u}\right)\left(., t_{j}\right) .
$$

Proposition 4.1 Assume that the velocity $\boldsymbol{u}$ of the solution $(\boldsymbol{u}, p)$ of problem (4.1)-(4.2) belongs to the space $H^{2}\left(0, \widetilde{T} ; H^{1}(\Omega)^{d}\right)$. Then, the following a priori error estimates holds for $1 \leqslant j \leqslant J$,

$$
\begin{gathered}
\left\|\boldsymbol{u}\left(., t_{j}\right)-\boldsymbol{u}^{j}\right\|_{H^{1}(\Omega)^{d}} \leqslant \frac{1}{\sqrt{3 c \nu_{1}}}|\tau|\|\boldsymbol{u}\|_{H^{2}\left(0, t_{j} ; H^{1}(\Omega)^{d}\right)}, \\
\left(\sum_{m=1}^{j} \tau_{m}\left\|\frac{\left(\boldsymbol{u}\left(., t_{m}\right)-\boldsymbol{u}^{m}\right)-\left(\boldsymbol{u}\left(., t_{m-1}\right)-\boldsymbol{u}^{m-1}\right)}{\tau_{m}}\right\|_{L^{2}(\Omega)^{d}}^{2}\right)^{\frac{1}{2}} \leqslant \frac{|\tau|}{\sqrt{3 \nu_{1}}}\|\boldsymbol{u}\|_{H^{2}\left(0, t_{j} ; H^{1}(\Omega)^{d}\right)},
\end{gathered}
$$




$$
\left(\sum_{m=1}^{j} \tau_{m}\left\|p\left(., t_{m}\right)-p^{m}\right\|_{L^{2}(\Omega)}\right)^{\frac{1}{2}} \leqslant \frac{1}{\sqrt{3 c \nu_{1}}}|\tau|\|\boldsymbol{u}\|_{H^{2}\left(0, t_{j} ; H^{1}(\Omega)^{d}\right)} .
$$

Proof: 1) Applying the same arguments as for of (3.9) to problem (4.12) yield

$$
\left\|\boldsymbol{e}_{\boldsymbol{u}}^{j}\right\|_{H^{1}(\Omega)^{d}}^{2} \leqslant \frac{1}{c \nu_{1}} \sum_{m=1}^{j} \tau_{m}\left\|\varepsilon_{\boldsymbol{u}}^{m}\right\|_{H^{-1}(\Omega)^{d}}^{2} .
$$

In order to estimate $\varepsilon_{\boldsymbol{u}}^{m}$, we use Taylor's expansion:

$$
\boldsymbol{u}\left(., t_{j}\right)-\boldsymbol{u}\left(., t_{j-1}\right)=\tau_{j}\left(\partial_{t} \boldsymbol{u}\right)\left(., t_{j}\right)-\int_{t_{j-1}}^{t_{j}}\left(t-t_{j-1}\right)\left(\partial_{t t}^{2} \boldsymbol{u}\right)(., t) d t
$$

whence

$$
\varepsilon_{\boldsymbol{u}}^{j}=-\frac{1}{\tau_{j}} \int_{t_{j-1}}^{t_{j}}\left(t-t_{j-1}\right)\left(\partial_{t t}^{2} \boldsymbol{u}\right)(., t) d t .
$$

A Cauchy-Schwarz inequality in this formula gives

$$
\left\|\varepsilon_{\boldsymbol{u}}^{j}\right\|_{L^{2}(\Omega)^{d}} \leqslant \frac{1}{\sqrt{3}} \tau_{j}^{\frac{1}{2}}\|\boldsymbol{u}\|_{H^{2}\left(t_{j-1}, t_{j} ; H^{1}(\Omega)^{d}\right)}
$$

The desired estimate is obtained by substituting this bound into (4.17).

2) By taking $\boldsymbol{v}$ equal to $\boldsymbol{e}_{\boldsymbol{u}}^{j}-\boldsymbol{e}_{\boldsymbol{u}}^{j-1}$ in (4.12) and recalling that $\boldsymbol{e}_{\boldsymbol{u}}^{0}$ is zero and $\left(\operatorname{div} \boldsymbol{v}, p\left(., t_{j}\right)-p^{j}\right)$ vanishes on $\Omega$, lead in particular to

$$
\sum_{m=1}^{j} \tau_{m}\left\|\frac{\left(\boldsymbol{u}\left(., t_{m}\right)-\boldsymbol{u}^{m}\right)-\left(\boldsymbol{u}\left(., t_{m-1}\right)-\boldsymbol{u}^{m-1}\right)}{\tau_{m}}\right\|_{L^{2}(\Omega)^{d}}^{2} \leqslant \frac{1}{\nu_{1}} \sum_{m=1}^{j} \tau_{m}\left\|\varepsilon_{\boldsymbol{u}}^{m}\right\|_{L^{2}(\Omega)^{d}}^{2},
$$

and by using (4.18) we obtain the desired result.

3) To prove the third inequality (4.16) we derive from the error equation (4.12) that

$$
\forall \boldsymbol{v} \in H_{0}^{1}(\Omega)^{d}, \quad-\left(\operatorname{div} \boldsymbol{v}, p\left(., t_{j}\right)-p^{j}\right)=-\left(\frac{\boldsymbol{e}_{\boldsymbol{u}}^{j-1}-\boldsymbol{e}_{\boldsymbol{u}}^{j}}{\tau_{j}}, \boldsymbol{v}\right)-\left(\nu(\theta) \nabla \boldsymbol{e}_{\boldsymbol{u}}^{j}, \nabla \boldsymbol{v}\right)+\left\langle\varepsilon_{\boldsymbol{u}}^{j}, \boldsymbol{v}\right\rangle_{\Omega} .
$$

We use inf-sup condition (2.8). Then this equation implies

$$
\left\|p\left(., t_{j}\right)-p^{j}\right\|_{L^{2}(\Omega)} \leqslant\left\|\frac{\boldsymbol{e}_{\boldsymbol{u}}^{j-1}-\boldsymbol{e}_{\boldsymbol{u}}^{j}}{\tau_{j}}\right\|_{L^{2}(\Omega)^{d}}+c \nu_{2}\left\|\boldsymbol{e}_{\boldsymbol{u}}^{j}\right\|_{H^{1}(\Omega)^{d}}+\left\|\varepsilon_{\boldsymbol{u}}^{j}\right\|_{L^{2}(\Omega)^{d}},
$$

and (4.16) follows by multiplying the square of this inequality bu $\tau_{j}$, summing over $j$ and using (4.19) and (4.18).

Similar but simpler arguments lead to

Proposition 4.2 Assume that the temperature $T$ of problem (4.3)-(4.4) belongs to the space $H^{2}\left(0, \widetilde{T} ; H^{1}(\Omega)\right)$ and the data $T_{b}$ belongs to $H^{2}\left(0, \widetilde{T} ; H^{\frac{1}{2}}(\partial \Omega)\right)$. Then, the following a priori error estimates holds for $1 \leqslant j \leqslant J$,

$$
\left\|T\left(., t_{j}\right)-T^{j}\right\|_{H^{1}(\Omega)} \leqslant \frac{|\tau|}{\sqrt{3 c}}\left(\|T\|_{H^{2}\left(0, t_{j} ; H^{1}(\Omega)\right)}+\left\|T_{b}\right\|_{H^{2}\left(0, t_{j} ; H^{\frac{1}{2}}(\partial \Omega)\right)}\right),
$$


We end this paragraph with the basic properties of the semi-discrete operators $\mathcal{S}_{\tau}(\theta)$ and $\mathcal{L}_{\tau}$. The operator $\mathcal{S}_{\tau}(\theta)$ satisfies the following three properties

(i) Stability: For any data $\boldsymbol{F}$ in $\mathscr{C}^{0}\left(0, \widetilde{T} ; H^{-1}(\Omega)^{d}\right)$,

$$
\left\|\mathcal{S}_{\tau}(\theta)(\boldsymbol{F}, 0)\right\|_{L^{2}\left(0, \widetilde{T} ; H^{1}(\Omega)^{d}\right)} \leqslant c\left\|\pi_{\tau}^{+} \boldsymbol{F}\right\|_{L^{2}\left(0, \widetilde{T} ; H^{-1}(\Omega)^{d}\right)}
$$

(ii) A priori error estimate: For any data $F$ in $\mathscr{C}^{0}\left(0, \widetilde{T} ; H^{-1}(\Omega)^{d}\right)$

$$
\left\|\left(\mathcal{S}-\mathcal{S}_{\tau}\right)(\theta)\left(F, \boldsymbol{u}_{0}\right)\right\|_{L^{2}\left(0, \widetilde{T} ; H^{1}(\Omega)^{d}\right)} \leqslant c|\tau|\left\|\mathcal{S}(\theta)\left(\boldsymbol{F}, \boldsymbol{u}_{0}\right)\right\|_{H^{2}\left(0, \widetilde{T} ; H^{1}(\Omega)^{d}\right)} .
$$

(iii) Convergence: For any data $\boldsymbol{F}$ in $\mathscr{C}^{0}\left(0, \widetilde{T} ; H^{-1}(\Omega)^{d}\right)$,

$$
\lim _{|\tau| \rightarrow 0}\left\|\left(\mathcal{S}-\mathcal{S}_{\tau}\right)(\theta)(\boldsymbol{F}, 0)\right\|_{L^{2}\left(0, \widetilde{T} ; H^{1}(\Omega)^{d}\right)}=0 .
$$

The analogous properties concerning the semi discrete operator $\mathcal{L}_{\tau}$ read :

(i) Stability: For any $G$ in $\mathscr{C}^{0}\left(0, \widetilde{T} ; H^{-1}(\Omega)\right)$

$$
\left\|\mathcal{L}_{\tau}(G, 0,0)\right\|_{L^{2}\left(0, \widetilde{T} ; H^{1}(\Omega)\right)} \leqslant c\left\|\pi_{\tau}^{+} G\right\|_{L^{2}\left(0, \widetilde{T} ; H^{-1}(\Omega)\right)}
$$

(ii) A priori error estimate: For any data $G$ in $\mathscr{C}^{0}\left(0, \widetilde{T} ; H^{-1}(\Omega)\right)$

$$
\left\|\left(\mathcal{L}-\mathcal{L}_{\tau}\right)\left(G, T_{b}, T_{0}\right)\right\|_{L^{2}\left(0, \widetilde{T} ; H^{1}(\Omega)\right)} \leqslant c|\tau|\left(\left\|\mathcal{L}\left(G, T_{b}, T_{0}\right)\right\|_{H^{2}\left(0, \widetilde{T} ; H^{1}(\Omega)\right)}+\left\|T_{b}\right\|_{H^{2}\left(0, \widetilde{T} ; H^{\frac{1}{2}}(\partial \Omega)\right)}\right)
$$

(iii) Convergence: For any data $G$ in $\mathscr{C}^{0}\left(0, \widetilde{T} ; H^{-1}(\Omega)\right)$,

$$
\lim _{|\tau| \rightarrow 0}\left\|\left(\mathcal{L}-\mathcal{L}_{\tau}\right)(G, 0,0)\right\|_{L^{2}\left(0, \widetilde{T} ; H^{1}(\Omega)\right)}=0 .
$$

\subsubsection{About the space discretization}

Similarly, we denote by $\boldsymbol{u}_{N \tau}$ and $T_{N \tau}$ the functions which are affine on each interval $\left[t_{j-1}, t_{j}\right]$ and equal to $\boldsymbol{u}_{N}^{j}$ and $T_{N}^{j}$ respectively at each time $t_{j}, 0 \leqslant j \leqslant J$, and also by $p_{N \tau}$ the function which is piecewise constant and equal to $p_{N}^{j}$ on each interval $\left.] t_{j-1}, t_{j}\right], 1 \leqslant j \leqslant J$. We also define $\mathcal{S}_{N \tau}(\theta)$ the discrete Stokes operator, i.e., the operator which associates with any data $\boldsymbol{F}$ continuous on $\bar{\Omega} \times[0, \widetilde{T}]$ and $\boldsymbol{u}_{0}$ in $H_{0}^{1}(\Omega)^{d}$, the part $\boldsymbol{u}_{N \tau}$ of the solution $\left(\boldsymbol{u}_{N}^{j}, p_{N}^{j}\right)$ of the Stokes problem

Find $\left(\boldsymbol{u}_{N}^{j}\right)_{0 \leqslant j \leqslant J}$ in $\mathbb{X}_{N}^{J+1}$ and $\left(p_{N}^{j}\right)_{1 \leqslant j \leqslant J}$ in $\mathbb{M}_{N}^{J}$ such that

$$
\boldsymbol{u}_{N}^{j}=\mathbf{0} \quad \text { on } \partial \Omega, 1 \leqslant j \leqslant J \quad \text { and } \quad \boldsymbol{u}_{N}^{0}=\mathcal{I}_{N} \boldsymbol{u}_{0} \text { in } \Omega,
$$

and, for $1 \leqslant j \leqslant J$,

$$
\begin{aligned}
& \forall \boldsymbol{v}_{N} \in \mathbb{X}_{N}, \quad\left(\frac{\boldsymbol{u}_{N}^{j}-\boldsymbol{u}_{N}^{j-1}}{\tau_{j}}, \boldsymbol{v}_{N}\right)_{N}+\left(\nu(\theta) \nabla \boldsymbol{u}_{N}^{j}, \nabla \boldsymbol{v}_{N}\right)_{N}-\left(\operatorname{div} \boldsymbol{v}_{N}, p_{N}^{j}\right)_{N}=\left\langle\boldsymbol{F}\left(., t_{j}\right), \boldsymbol{v}_{N}\right\rangle_{\Omega}, \\
& \forall q_{N} \in \mathbb{M}_{N}, \quad-\left(\operatorname{div} \boldsymbol{u}_{N}^{j}, q_{N}\right)_{N}=0 .
\end{aligned}
$$

Let finally $\mathcal{L}_{N \tau}$ denote the operator which associates with any datum $G, T_{b}$ continuous on $\bar{\Omega} \times[0, \widetilde{T}]$ and $T_{0}$ in $H^{1}(\Omega)$,

$$
\begin{gathered}
T_{N}^{j}=i_{N}^{\partial \Omega} T_{b}\left(., t_{j}\right) \quad \text { on } \partial \Omega, 1 \leqslant j \leqslant J \quad \text { and } \quad T_{N}^{0}=\mathcal{I}_{N} T_{0} \quad \operatorname{in} \Omega, \\
\forall S_{N} \in \mathbb{Y}_{N}^{0}, \quad\left(\frac{T_{N}^{j}-T_{N}^{j-1}}{\tau_{j}}, S_{N}\right)_{N}+\alpha\left(\operatorname{grad} T_{N}^{j}, \operatorname{grad} S_{N}\right)_{N}=\left\langle G\left(., t_{j}\right), S_{N}\right\rangle_{\Omega}
\end{gathered}
$$


We are in position to state some properties of the operator $\mathcal{S}_{N \tau}(\theta)$, the a priori error estimate (ii) in the following properties being derived by choosing for any divergence-free polynomial $\boldsymbol{v}_{N}$ in $\mathbb{X}_{N-1}$, $\boldsymbol{u}_{N}^{j}-\boldsymbol{v}_{N}^{j}$ as a function test in (4.28). Then from standard arguments in spectral methods, (see [6, Chap. V] for instance), we derive

(i) Stability: For any datum $\boldsymbol{F}$ in $\mathscr{C}^{0}\left(0, \widetilde{T} ; H^{-1}(\Omega)^{d}\right)$,

$$
\left\|\mathcal{S}_{N \tau}(\theta)(\boldsymbol{F}, 0)\right\|_{L^{2}\left(0, \widetilde{T} ; H^{1}(\Omega)^{d}\right)} \leqslant c \sum_{m=1}^{j} \tau_{m} \sup _{\boldsymbol{v}_{N} \in \mathbb{X}_{N}} \frac{\int_{\Omega} \boldsymbol{F}\left(\boldsymbol{x}, t_{m}\right) \boldsymbol{v}_{N}\left(\boldsymbol{x}, t_{m}\right) \mathrm{d} \boldsymbol{x}}{\left\|\boldsymbol{v}_{N}\right\|_{H^{1}(\Omega)^{d}}},
$$

(ii) A priori error estimate: If moreover $\widetilde{\mathcal{S}_{\tau}}(\theta)\left(\boldsymbol{F}, \boldsymbol{u}_{0}\right)$ belongs to $L^{2}\left(0, \widetilde{T} ; H^{s-1}(\Omega)\right)$ and $\mathcal{S}_{\tau}(\theta)\left(\boldsymbol{F}, \boldsymbol{u}_{0}\right)$ to $L^{2}\left(0, \widetilde{T} ; H^{s}(\Omega)^{d}\right)$ for a real number $s, s \geqslant 1$,

$$
\begin{aligned}
& \left\|\left(\mathcal{S}_{\tau}-\mathcal{S}_{N \tau}\right)(\theta)\left(\boldsymbol{F}, \boldsymbol{u}_{0}\right)\right\|_{L^{2}\left(0, \widetilde{T} ; H^{1}(\Omega)^{d}\right)} \leqslant c N^{1-s}\left(\left\|\mathcal{S}_{\tau}(\theta)\left(\boldsymbol{F}, \boldsymbol{u}_{0}\right)\right\|_{L^{2}\left(0, \widetilde{T} ; H^{s}(\Omega)^{d}\right)}\right. \\
& \left.+\left\|\widetilde{\mathcal{S}_{\tau}}(\theta)\left(\boldsymbol{F}, \boldsymbol{u}_{0}\right)\right\|_{L^{2}\left(0, \widetilde{T} ; H^{s-1}(\Omega)\right)}\right) .
\end{aligned}
$$

(iii) Convergence: For any data $\boldsymbol{F}$ in $\mathscr{C}^{0}\left(0, \widetilde{T} ; H^{-1}(\Omega)^{d}\right)$,

$$
\lim _{N \rightarrow+\infty}\left\|\left(\mathcal{S}_{\tau}-\mathcal{S}_{N \tau}\right)(\theta)(\boldsymbol{F}, 0)\right\|_{L^{2}\left(0, \widetilde{T} ; H^{1}(\Omega)^{d}\right)}=0 .
$$

The analogous properties concerning the discrete operator $\mathcal{L}_{N \tau}$ and the operator $\mathcal{L}_{\tau}$ read (i) Stability: For any $G$ in $\mathscr{C}^{0}\left(0, \widetilde{T} ; H^{-1}(\Omega)\right)$

$$
\left\|\mathcal{L}_{N \tau}(G, 0,0)\right\|_{L^{2}\left(0, \widetilde{T} ; H^{1}(\Omega)\right)} \leqslant c \sum_{m=1}^{j} \tau_{m} \sup _{S_{N} \in \mathbb{Y}_{N}^{0}} \frac{\int_{\Omega} G\left(\boldsymbol{x}, t_{m}\right) S_{N}\left(\boldsymbol{x}, t_{m}\right) \mathrm{d} \boldsymbol{x}}{\left\|S_{N}\right\|_{H^{1}(\Omega)}} .
$$

(ii) A priori error estimate: If moreover $\mathcal{L}_{\tau}\left(G, T_{b}, T_{0}\right)$ belongs to $L^{2}\left(0, \widetilde{T} ; H^{s}(\Omega)\right), s \geqslant 1$ and $T_{b}$ belongs to $L^{2}\left(0, \widetilde{T} ; H^{\sigma}(\partial \Omega)\right)$, for a real number $\sigma, \sigma>\frac{d-1}{2}$,

$$
\begin{aligned}
\left\|\left(\mathcal{L}_{\tau}-\mathcal{L}_{N \tau}\right)\left(G, T_{b}, T_{0}\right)\right\|_{L^{2}\left(0, \widetilde{T} ; H^{1}(\Omega)\right)} \leqslant c N^{1-s}\left\|\mathcal{L}_{\tau}\left(G, T_{b}, T_{0}\right)\right\|_{L^{2}\left(0, \widetilde{T} ; H^{s}(\Omega)\right)} \\
+N^{\frac{1}{2}-\sigma}\left\|T_{b}\right\|_{L^{2}\left(0, \widetilde{T} ; H^{\sigma}(\partial \Omega)\right)} .
\end{aligned}
$$

(iii) Convergence: For any data $G$ in $\mathscr{C}^{0}\left(0, \widetilde{T} ; H^{-1}(\Omega)\right)$,

$$
\lim _{N \rightarrow+\infty}\left\|\left(\mathcal{L}_{\tau}-\mathcal{L}_{N \tau}\right)(G, 0,0)\right\|_{L^{2}\left(0, \widetilde{T} ; H^{1}(\Omega)\right)}=0 .
$$

To conclude, with the notation $U_{N \tau}=\left(\boldsymbol{u}_{N \tau}, T_{N \tau}\right)$, problem (3.13)-(3.14)-(3.15) can equivalently be written as

$$
\begin{array}{r}
\mathcal{F}_{N \tau}\left(U_{N \tau}\right)=U_{N \tau}-\left(\begin{array}{cc}
\mathcal{S}_{N \tau}\left(\pi_{\tau}^{-} T_{N \tau}\right) & 0 \\
0 & \mathcal{L}_{N \tau}
\end{array}\right) \mathcal{G}_{N \tau}\left(U_{N \tau}\right)=0 \\
\text { with } \\
\mathcal{G}_{N \tau}\left(U_{N \tau}\right)=\left(\begin{array}{c}
\left(\mathcal{G}_{N \tau 1}, \boldsymbol{u}_{0}\right) \\
\left(\mathcal{G}_{N \tau 2}, T_{b}, T_{0}\right)
\end{array}\right) .
\end{array}
$$

The two components $\mathcal{G}_{N 1 \tau}$ and $\mathcal{G}_{N 2 \tau}$ are defined by

$$
\begin{aligned}
\forall \boldsymbol{v}_{N} \in \mathbb{X}_{N}, \quad \int_{\Omega} \mathcal{G}_{N \tau 1}(\boldsymbol{x}, t) \cdot \boldsymbol{v}_{N}(\boldsymbol{x}) d \boldsymbol{x}=\left(\boldsymbol{f}-\left(\boldsymbol{u}_{N \tau} \cdot \nabla\right) \boldsymbol{u}_{N \tau}, \boldsymbol{v}_{N}\right)_{N} \\
\forall S_{N} \in \mathbb{Y}_{N}^{0}, \quad \int_{\Omega} \mathcal{G}_{N \tau 2}(\boldsymbol{x}, t) . S_{N}(\boldsymbol{x}) d \boldsymbol{x}=\left(g-\left(\boldsymbol{u}_{N \tau} \cdot \nabla\right) T_{N \tau}, S_{N}\right)_{N} .
\end{aligned}
$$


From now on, we denote by

$$
\mathbb{Z}=L^{2}\left(0, \widetilde{T} ; H_{0}^{1}(\Omega)^{d}\right) \times L^{2}\left(0, \widetilde{T} ; H^{1}(\Omega)\right) .
$$

We also introduce the fully discrete space, i.e, the space $\mathbb{Z}_{N \tau}$ of functions which are affine on each interval $\left[t_{j-1}, t_{j}\right], 1 \leqslant j \leqslant J$, and such that their values in $t_{j}$ belong to $\mathbb{X}_{N} \times \mathbb{Y}_{N}$. It is readily checked that this space is finite-dimensional and imbedded in $\mathbb{Z}$.

To go further, we introduce an approximation $U_{N \tau}^{\diamond}=\left(\boldsymbol{u}_{N \tau}^{\diamond}, T_{N \tau}^{\diamond}\right)$ of the solution $U=(\boldsymbol{u}, T)$ in $\mathbb{Z}_{N \tau}$, for $0 \leqslant r \leqslant 1$

$$
\begin{aligned}
\left\|\left(\boldsymbol{u}-\boldsymbol{u}_{N \tau}^{\diamond}\right)(., t)\right\|_{H^{r}(\Omega)^{d}} \leqslant & c N^{r-1}\|\boldsymbol{u}(., t)\|_{H^{1}(\Omega)^{d}}, \\
& \left\|\left(T-T_{N \tau}^{\diamond}\right)(., t)\right\|_{H^{r}(\Omega)} \leqslant c N^{r-1}\|T(., t)\|_{H^{1}(\Omega)} .
\end{aligned}
$$

The existence of such an approximation is stated in [5, Thm 7.4] (see also [6, Chap. III, Thm. 2.4 \& Chap. VI, Thm. 2.5] ).

Lemma 4.3 If the data $\boldsymbol{f}$ belong to $L^{2}\left(0, \widetilde{T} ; H^{\sigma}(\Omega)^{d}\right), \sigma>\frac{d}{2}$, the following result holds for any $t \in] 0, \widetilde{T}[$

$\int_{0}^{t}\left\langle\mathcal{G}_{1}\left(\boldsymbol{u}_{N \tau}\right)-\mathcal{G}_{N \tau 1}\left(\boldsymbol{u}_{N \tau}\right), \boldsymbol{v}_{N}\right\rangle_{\Omega} d s \leqslant c\left(N^{-\frac{1}{2}}\left\|\boldsymbol{u}_{N \tau}\right\|_{L^{2}\left(0, \widetilde{T} ; H^{1}(\Omega)^{d}\right)}^{2}+N^{-\sigma}\|\boldsymbol{f}\|_{L^{2}\left(0, \widetilde{T} ; H^{\sigma}(\Omega)^{d}\right)}\right)\left\|\boldsymbol{v}_{N}\right\|_{H^{1}(\Omega)^{d}}$.

Proof: We have,

$$
\left\langle\mathcal{G}_{1}\left(\boldsymbol{u}_{N \tau}\right)-\mathcal{G}_{N \tau 1}\left(\boldsymbol{u}_{N \tau}\right), \boldsymbol{v}_{N}\right\rangle_{\Omega}=\left(\boldsymbol{f}-\left(\boldsymbol{u}_{N \tau} \cdot \nabla\right) \boldsymbol{u}_{N \tau}, \boldsymbol{v}_{N}\right)-\left(\boldsymbol{f}-\left(\boldsymbol{u}_{N \tau} \cdot \nabla\right) \boldsymbol{u}_{N \tau}, \boldsymbol{v}_{N}\right)_{N}
$$

If $N^{\prime}$ stands for the integer part of $\frac{N-1}{2}$, we introduce an approximation $\boldsymbol{u}_{N^{\prime}}$ of $\boldsymbol{u}_{N \tau}$ in $\mathbb{P}_{N^{\prime}}(\Omega)^{d}$ and we note the identity

$$
\left(\left(\boldsymbol{u}_{N^{\prime}} \cdot \nabla\right) \boldsymbol{u}_{N^{\prime}}, \boldsymbol{v}_{N}\right)=\left(\left(\boldsymbol{u}_{N^{\prime}} \cdot \nabla\right) \boldsymbol{u}_{N^{\prime}}, \boldsymbol{v}_{N}\right)_{N}
$$

Inserting it, we obtain

$$
\begin{aligned}
\left\langle\mathcal{G}_{1}\left(\boldsymbol{u}_{N \tau}\right)-\mathcal{G}_{N \tau 1}\left(\boldsymbol{u}_{N \tau}\right), \boldsymbol{v}_{N}\right\rangle_{\Omega}=( & \left(\boldsymbol{u}_{N^{\prime}} \cdot \nabla\right) \\
& +\left(\left(\boldsymbol{u}_{N \tau} \cdot \nabla\right) \boldsymbol{u}_{N \tau}-\left(\boldsymbol{u}_{N^{\prime}} \cdot \nabla\right) \boldsymbol{u}_{N^{\prime}}, \boldsymbol{v}_{N}\right)_{N} \\
& +\left(\left(\boldsymbol{f}, \boldsymbol{v}_{N}\right)-\left(\mathcal{I}_{N} \boldsymbol{f}, \boldsymbol{v}_{N}\right)_{N}\right) .
\end{aligned}
$$

The reasons to evaluating the first two quantities are the same, so we only consider the first one

$$
\left(\left(\boldsymbol{u}_{N^{\prime}} \cdot \nabla\right) \boldsymbol{u}_{N^{\prime}}-\left(\boldsymbol{u}_{N \tau} \cdot \nabla\right) \boldsymbol{u}_{N \tau}, \boldsymbol{v}_{N}\right)=\left(\left(\boldsymbol{u}_{N^{\prime}}-\boldsymbol{u}_{N \tau} \cdot \nabla\right) \boldsymbol{u}_{N \tau}, \boldsymbol{v}_{N}\right)+\left(\left(\boldsymbol{u}_{N^{\prime}} \cdot \nabla\right)\left(\boldsymbol{u}_{N^{\prime}}-\boldsymbol{u}_{N \tau}\right), \boldsymbol{v}_{N}\right)
$$

we obtain

$$
\begin{aligned}
\left(\left(\boldsymbol{u}_{N^{\prime}}-\boldsymbol{u}_{N \tau} \cdot \nabla\right) \boldsymbol{u}_{N \tau}, \boldsymbol{v}_{N}\right) & \leqslant\left\|\left(\boldsymbol{u}_{N \tau}-\boldsymbol{u}_{N^{\prime}}\right)(., t)\right\|_{L^{3}(\Omega)^{d}}\left\|\boldsymbol{v}_{N}\right\|_{L^{6}(\Omega)^{d}}\left\|\nabla \boldsymbol{u}_{N \tau}(., t)\right\|_{L^{2}(\Omega)^{d \times d}} \\
& \leqslant\left\|\left(\boldsymbol{u}_{N \tau}-\boldsymbol{u}_{N^{\prime}}\right)(., t)\right\|_{H^{\frac{1}{2}}(\Omega)^{d}}\left\|\boldsymbol{v}_{N}\right\|_{H^{1}(\Omega)^{d}}\left|\boldsymbol{u}_{N \tau}(., t)\right|_{H^{1}(\Omega)^{d}}
\end{aligned}
$$

we conclude by using (4.38) for $r=\frac{1}{2}$ and by integrating between 0 and $t$.

To evaluating the third term in (4.39), we have for any $\boldsymbol{f}_{N-1}$ in $\mathbb{P}_{N-1}(\Omega)^{d}$,

$$
\begin{gathered}
\left(\boldsymbol{f}, \boldsymbol{v}_{N}\right)-\left(\boldsymbol{f}, \boldsymbol{v}_{N}\right)_{N}=\int_{\Omega}\left(\boldsymbol{f}-\boldsymbol{f}_{N-1}\right)(\boldsymbol{x}, t) . \boldsymbol{v}_{N}(\boldsymbol{x}) d \boldsymbol{x}-\left(\boldsymbol{f}-\boldsymbol{f}_{N-1}, \boldsymbol{v}_{N}\right)_{N} \\
\left(\boldsymbol{f}, \boldsymbol{v}_{N}\right)-\left(\boldsymbol{f}, \boldsymbol{v}_{N}\right)_{N} \leqslant c\left(\left\|\left(\boldsymbol{f}-\mathcal{I}_{N} \boldsymbol{f}\right)(., t)\right\|_{L^{2}(\Omega)^{d}}+\inf _{\boldsymbol{f}_{N-1} \in \mathbb{P}_{N-1}(\Omega)}\left\|\left(\boldsymbol{f}-\boldsymbol{f}_{N-1}\right)(., t)\right\|_{L^{2}(\Omega)^{d}}\right)\left\|\boldsymbol{v}_{N}\right\|_{H^{1}(\Omega)^{d}}
\end{gathered}
$$

By taking $\boldsymbol{f}_{N-1}$ equal to $\Pi_{N-1} \boldsymbol{f}$, where $\Pi_{N-1}$ stands for the orthogonal projection operator from $L^{2}(\Omega)^{d}$ onto $\mathbb{P}_{N-1}(\Omega)^{d}$ (see [6, Chap. III]), using [6, Chap. IV, Thm 2.6] and [6, Chap. III, Thm 2.4], and by integrating between 0 and $t$ we obtain the desired result. 


\subsection{Some more lemmas}

We denote by $\mathcal{E}(\mathbb{Z})$ the space of endomorphisms of $\mathbb{Z}$ and we make the following assumption.

Assumption 4.4 The solution $U=(\boldsymbol{u}, T)$ of problem $(2.5)-(2.6)-(2.7)$

(i) is such that $D \mathcal{F}(U)$ is an isomorphism of $\mathbb{Z}$, where $D$ stands for the differential operator with respect to $U$,

(ii) belongs to $H^{1}\left(0, \widetilde{T} ; H^{\rho}(\Omega)^{d}\right) \times H^{1}\left(0, \widetilde{T} ; H^{\rho}(\Omega)\right), \rho>1$.

Lemma 4.5 Assume that $\nu$ is of class $\mathscr{C}^{2}$ on $\mathbb{R}$, with bounded derivatives, and Assumption 4.4 holds. There exists a positive integer $\tau_{0}$ and $N_{0}$ such that, for all $\tau,|\tau| \leqslant \tau_{0}$ and for all $N \geqslant N_{0}$, the operator $D \mathcal{F}_{N \tau}\left(U_{N \tau}^{\diamond}\right)$ is an isomorphism of $\mathbb{Z}_{N \tau}$, and the norm of its inverse is bounded independently of $\tau$ and $N$.

Proof: We write the expansion

$$
\begin{gathered}
D \mathcal{F}_{N \tau}\left(U_{N \tau}^{\diamond}\right)=D \mathcal{F}(U)-\left(\begin{array}{cc}
\mathcal{S}_{N \tau}\left(\pi_{\tau}^{-} T_{N \tau}^{\diamond}\right)-\mathcal{S}(T) & 0 \\
0 & \mathcal{L}_{N \tau}-\mathcal{L}
\end{array}\right) D \mathcal{G}(U) \\
-\left(\begin{array}{cc}
\mathcal{S}_{N \tau}\left(\pi_{\tau}^{-} T_{N \tau}^{\diamond}\right) & 0 \\
0 & \mathcal{L}_{N \tau}
\end{array}\right)\left(D \mathcal{G}\left(U_{N \tau}^{\diamond}\right)-D \mathcal{G}(U)\right)-\left(\begin{array}{cc}
\mathcal{S}_{N \tau}\left(\pi_{\tau}^{-} T_{N \tau}^{\diamond}\right) & 0 \\
0 & \mathcal{L}_{N \tau}
\end{array}\right)\left(D \mathcal{G}_{N \tau}\left(U_{N \tau}^{\diamond}\right)-D \mathcal{G}\left(U_{N \tau}^{\diamond}\right)\right) \\
-\left(\begin{array}{cc}
D \mathcal{S}_{N \tau}\left(\pi_{\tau}^{-} T_{N \tau}^{\diamond}\right)-D \mathcal{S}(T) & 0 \\
0 & 0
\end{array}\right) \mathcal{G}(U)-\left(\begin{array}{cc}
D \mathcal{S}_{N \tau}\left(\pi_{\tau}^{-} T_{N \tau}^{\diamond}\right) & 0 \\
0 & 0
\end{array}\right)\left(\mathcal{G}\left(U_{N \tau}^{\diamond}\right)-\mathcal{G}(U)\right) \\
-\left(\begin{array}{cc}
D \mathcal{S}_{N \tau}\left(\pi_{\tau}^{-} T_{N \tau}^{\diamond}\right) & 0 \\
0 & 0
\end{array}\right)\left(\mathcal{G}_{N \tau}\left(U_{N \tau}^{\diamond}\right)-\mathcal{G}\left(U_{N \tau}^{\diamond}\right)\right)
\end{gathered}
$$

Due to part (i) of Assumption 4.4, we only have to check that the last six terms in the right-hand side tend to zero when $|\tau|$ and $N^{-1}$ tend to zero in the norm of the space $\mathcal{E}(\mathbb{Z})$. Let $W_{N \tau}=\left(\boldsymbol{w}_{N \tau}, R_{N \tau}\right)$ be any element in the unit sphere of $\mathbb{Z}_{N \tau}$.

1) We observe that

$$
D \mathcal{G}(U) . W_{N \tau}=\left(\begin{array}{c}
-\left((\boldsymbol{u} \cdot \nabla) \boldsymbol{w}_{N \tau}+\left(\boldsymbol{w}_{N \tau} \cdot \nabla\right) \boldsymbol{u}, 0\right) \\
-\left((\boldsymbol{u} \cdot \nabla) R_{N \tau}+\left(\boldsymbol{w}_{N \tau} \cdot \nabla\right) T, 0,0\right)
\end{array}\right)
$$

Since $\mathbb{Z}_{N \tau}$ is finite-dimensional, we deduce from (ii) of Assumption 4.4, that both terms $(\boldsymbol{u} . \nabla) \boldsymbol{w}_{N \tau}+\left(\boldsymbol{w}_{N \tau} . \nabla\right) \boldsymbol{u}$ and $(\boldsymbol{u} . \nabla) R_{N \tau}+\left(\boldsymbol{w}_{N \tau} . \nabla\right) T$ runs through a compact of $\mathscr{C}^{0}\left(0, \widetilde{T} ; H^{-1}(\Omega)^{d}\right)$ and $\mathscr{C}^{0}\left(0, \widetilde{T} ; H^{-1}(\Omega)\right)$ respectively. Thus owing to the expansion $\left(\mathcal{S}_{N \tau}-\mathcal{S}\right)(\theta)=\left(\mathcal{S}_{N \tau}-\mathcal{S}_{\tau}\right)(\theta)+$ $\left(\mathcal{S}_{\tau}-\mathcal{S}\right)(\theta)$ and $\mathcal{L}-\mathcal{L}_{N \tau}=\left(\mathcal{L}-\mathcal{L}_{\tau}\right)+\left(\mathcal{L}_{\tau}-\mathcal{L}_{N \tau}\right)$, combining all this with with the continuty of the operator $\mathcal{S}(\theta),(4.38),(4.33),(4.36),(4.26)$ and (4.23) leads to

$$
\lim _{N \rightarrow+\infty} \lim _{|\tau| \rightarrow 0}\left\|\left(\begin{array}{cc}
\mathcal{S}_{N \tau}\left(\pi_{\tau}^{-} T_{N \tau}^{\diamond}\right)-\mathcal{S}(T) & 0 \\
0 & \mathcal{L}_{N \tau}-\mathcal{L}
\end{array}\right) D \mathcal{G}(U)\right\|_{\mathcal{E}(\mathbb{Z})}=0 .
$$

2) Due to the definition of $D \mathcal{G}$, we must now proof the convergence of the two terms

$$
\begin{aligned}
& \left(\left(\boldsymbol{u}-\boldsymbol{u}_{N \tau}^{\diamond}\right) . \nabla\right) \boldsymbol{w}_{N \tau}+\left(\boldsymbol{w}_{N \tau} \cdot \nabla\right)\left(\boldsymbol{u}-\boldsymbol{u}_{N \tau}^{\diamond}\right), \\
& \left(\left(\boldsymbol{u}-\boldsymbol{u}_{N \tau}^{\diamond}\right) . \nabla\right) R_{N \tau}+\left(\boldsymbol{w}_{N \tau} \cdot \nabla\right)\left(T-T_{N \tau}^{\diamond}\right)
\end{aligned}
$$

Owing to (4.38) with combining (4.31) and (4.34), we obtain

$$
\lim _{N \rightarrow+\infty} \lim _{|\tau| \rightarrow 0}\left\|\left(\begin{array}{cc}
\mathcal{S}_{N \tau}\left(\pi_{\tau}^{-} T_{N \tau}^{\diamond}\right) & 0 \\
0 & \mathcal{L}_{N \tau}
\end{array}\right)\left(D \mathcal{G}\left(U_{N \tau}^{\diamond}\right)-D \mathcal{G}(U)\right)\right\|_{\mathcal{E}(\mathbb{Z})}=0 .
$$


3) Due the definition of $D \mathcal{G}$ and $D \mathcal{G}_{N \tau}$, we have

$$
\begin{aligned}
& \left\langle D \mathcal{G}_{N \tau}\left(U_{N \tau}^{\diamond}\right)-D \mathcal{G}\left(U_{N \tau}^{\diamond}\right), \boldsymbol{v}_{N}\right\rangle \cdot W_{N \tau} \\
& \quad=\left(\begin{array}{c}
\left.\left(\left(\boldsymbol{u}_{N \tau}^{\diamond} \cdot \nabla\right) \boldsymbol{w}_{N \tau}+\left(\boldsymbol{w}_{N \tau} \cdot \nabla\right) \boldsymbol{u}_{N \tau}^{\diamond}, \boldsymbol{v}_{N}\right)-\left(\left(\boldsymbol{u}_{N \tau}^{\diamond} \cdot \nabla\right) \boldsymbol{w}_{N \tau}+\left(\boldsymbol{w}_{N \tau} \cdot \nabla\right) \boldsymbol{u}_{N \tau}^{\diamond}, \boldsymbol{v}_{N}\right)_{N}, 0\right) \\
\left(\left(\left(\boldsymbol{u}_{N \tau}^{\diamond} \cdot \nabla\right) R_{N \tau}+\left(\boldsymbol{w}_{N \tau} \cdot \nabla\right) T_{N \tau}^{\diamond}\right)-\left(\left(\boldsymbol{u}_{N \tau}^{\diamond} \cdot \nabla\right) R_{N \tau}+\left(\boldsymbol{w}_{N \tau} \cdot \nabla\right) T_{N \tau}^{\diamond}\right)_{N}, 0,0\right)
\end{array}\right) .
\end{aligned}
$$

Since the arguments for evaluating the two terms are similar, we only consider the first one, we set $N^{\prime}$ equal to the integer part of $\frac{N-1}{2}$, and we introduce the approximation $\boldsymbol{u}_{N^{\prime}}$ of $\boldsymbol{u}_{N \tau}^{\diamond}$ in $\mathbb{P}_{N^{\prime}}(\Omega)^{d}$, by the same arguments in Lemma 4.3, we deduce

$$
\left\langle D \mathcal{G}_{N \tau}\left(U_{N \tau}^{\diamond}\right)-D \mathcal{G}\left(U_{N \tau}^{\diamond}\right), \boldsymbol{v}_{N}\right\rangle \cdot W_{N \tau} \leqslant c N^{-\frac{1}{2}}\left\|\boldsymbol{u}_{N \tau}^{\diamond}\right\|_{L^{2}\left(0, \widetilde{T} ; H^{1}(\Omega)^{d}\right)}^{2}\left\|\boldsymbol{v}_{N}\right\|_{H^{1}(\Omega)^{d}} .
$$

Owing to (4.31) and (4.34), thus yields

$$
\lim _{N \rightarrow+\infty} \lim _{|\tau| \rightarrow 0}\left\|\left(\begin{array}{cc}
\mathcal{S}_{N \tau}\left(\pi_{\tau}^{-} T_{N \tau}^{\diamond}\right) & 0 \\
0 & \mathcal{L}_{N \tau}
\end{array}\right)\left(D \mathcal{G}_{N \tau}\left(U_{N \tau}^{\diamond}\right)-D \mathcal{G}\left(U_{N \tau}^{\diamond}\right)\right)\right\|_{\mathcal{E}(\mathbb{Z})}=0
$$

4) On the other hand, we note that, for any $\boldsymbol{F}$ in $H^{-1}(\Omega)^{d}$,

$$
\begin{aligned}
\left(D \mathcal{S}(T) R_{N \tau}\right)\left(\boldsymbol{F}, \boldsymbol{u}_{0}\right) & =\mathcal{S}(T)\left(-\operatorname{div}\left(\partial_{\theta} \nu(T) R_{N \tau} \nabla \mathcal{S}(T)\left(\boldsymbol{F}, \boldsymbol{u}_{0}\right)\right)\right) \\
\left(D \mathcal{S}_{N \tau}\left(\pi_{\tau}^{-} T_{N \tau}^{\diamond}\right) R_{N \tau}\right)\left(\boldsymbol{F}, \boldsymbol{u}_{0}\right) & =\mathcal{S}_{N \tau}\left(\pi_{\tau}^{-} T_{N \tau}^{\diamond}\right)\left(-\operatorname{div}\left(\partial_{\theta} \nu\left(T_{N \tau}^{\diamond}\right) R_{N \tau} \nabla \mathcal{S}_{N \tau}\left(\pi_{\tau}^{-} T_{N \tau}^{\diamond}\right)\left(\boldsymbol{F}, \boldsymbol{u}_{0}\right)\right)\right)
\end{aligned}
$$

By subtracting the second line from the first one, we derive

$$
\begin{aligned}
& \left(\left(D \mathcal{S}_{N \tau}\left(\pi_{\tau}^{-} T_{N \tau}^{\diamond}\right)-D \mathcal{S}(T)\right) R_{N \tau}\right)\left(\boldsymbol{F}, \boldsymbol{u}_{0}\right)= \\
& \left(\mathcal{S}_{N \tau}\left(\pi_{\tau}^{-} T_{N \tau}^{\diamond}\right)-\mathcal{S}(T)\right)\left(-\operatorname{div}\left(\partial_{\theta} \nu(T) R_{N \tau} \nabla \mathcal{S}(T)\left(\boldsymbol{F}, \boldsymbol{u}_{0}\right)\right)\right) \\
& -\mathcal{S}_{N \tau}\left(\pi_{\tau}^{-} T_{N \tau}^{\diamond}\right)\left(-\operatorname{div}\left(\partial_{\theta} \nu\left(T_{N \tau}^{\diamond}\right) R_{N \tau} \nabla\left(\mathcal{S}-\mathcal{S}_{N \tau}\right)\left(\pi_{\tau}^{-} T_{N \tau}^{\diamond}\right)\left(\boldsymbol{F}, \boldsymbol{u}_{0}\right)\right)\right) \\
& \quad+\mathcal{S}_{N \tau}\left(\pi_{\tau}^{-} T_{N \tau}^{\diamond}\right)\left(-\operatorname{div}\left(\partial_{\theta} \nu\left(T_{N \tau}^{\diamond}\right) R_{N \tau} \nabla\left(\mathcal{S}\left(\pi_{\tau}^{-} T_{N \tau}^{\diamond}\right)-\mathcal{S}(T)\right)\left(\boldsymbol{F}, \boldsymbol{u}_{0}\right)\right)\right) \\
& \quad+\mathcal{S}_{N \tau}\left(\pi_{\tau}^{-} T_{N \tau}^{\diamond}\right)\left(-\operatorname{div}\left(\partial_{\theta}\left(\nu\left(T_{N \tau}^{\diamond}\right)-\nu(T)\right) R_{N \tau} \nabla \mathcal{S}(T)\left(\boldsymbol{F}, \boldsymbol{u}_{0}\right)\right)\right) .
\end{aligned}
$$

Denoting by $\left(\boldsymbol{F}, \boldsymbol{u}_{0}\right)$ the first component of $\mathcal{G}(U)$, we see that $\mathcal{S}(T)\left(\boldsymbol{F}, \boldsymbol{u}_{0}\right)$ is equal to $\boldsymbol{u}$, see (4.5). We deduce from the regularity assumption on $\boldsymbol{u}$, when $W_{N \tau}$ runs through unit sphere of $\mathbb{Z}_{N \tau}$ quantity $-\operatorname{div}\left(\partial_{\theta} \nu(T) R_{N \tau} \nabla \mathcal{S}(T)\left(\boldsymbol{F}, \boldsymbol{u}_{0}\right)\right)$ belongs to a compact subset of $\mathscr{C}^{0}\left(0, \widetilde{T} ; H^{-1}(\Omega)^{d}\right)$. Thus, the convergence of the first term to zero follows from with the continuty of the operator $\mathcal{S}(\theta),(4.23),(4.33)$ and (4.38), the convergence of the second term, follows from (4.31), (4.38), (4.22) and (4.32). The convergence of the third term is deduced from (4.31) and (4.38) and of the fourth term from (4.31), the bounded derivatives of $\nu$ and (4.38). Thus, we derive

$$
\lim _{|\tau| \rightarrow 0} \lim _{N \rightarrow+\infty}\left\|\left(\begin{array}{cc}
D \mathcal{S}_{N \tau}\left(\pi_{\tau}^{-} T_{N \tau}^{\diamond}\right)-D \mathcal{S}(T) & 0 \\
0 & 0
\end{array}\right) \mathcal{G}(U)\right\|_{\mathcal{E}(\mathbb{Z})}=0
$$

5) The convergence of the fifth term is deduced from (4.38) and the stability of $D \mathcal{S}_{N \tau}(\theta)$ and the convergence of the last term is obtained with the same arguments as for Lemma 4.3.

This concludes the proof.

Lemma 4.6 If the function $\nu$ belongs to $W^{2, \infty}(\mathbb{R})$, with Lipschitz-continuous derivative, there exist a neighbourhood of $U_{N \tau}^{\diamond}$ in $\mathbb{Z}_{N \tau}$ and a positive constant $c$ such that the following Lipschitz property holds for any $\widetilde{U}_{N}$ in this neighbourhood,

$$
\left\|D \mathcal{F}_{N \tau}\left(U_{N \tau}^{\diamond}\right)-D \mathcal{F}_{N \tau}\left(\widetilde{U}_{N}\right)\right\|_{\mathcal{E}\left(\mathbb{Z}_{N \tau}\right)} \leqslant c\left\|U_{N \tau}^{\diamond}-\widetilde{U}_{N}\right\|_{\mathbb{Z}},
$$

where $\mathcal{E}\left(\mathbb{Z}_{N \tau}\right)$ stands for the space of endomorphisms of $\mathbb{Z}_{N \tau}$. 
Proof: Setting $\widetilde{U}_{N}=\left(\widetilde{\boldsymbol{u}}_{N}, \widetilde{T}_{N}\right)$, we have

$$
\begin{aligned}
D \mathcal{F}_{N \tau}\left(U_{N \tau}^{\diamond}\right)-D \mathcal{F}_{N \tau}\left(\widetilde{U}_{N}\right)=\left(\begin{array}{cc}
\mathcal{S}_{N \tau}\left(\pi_{\tau}^{-} \widetilde{T}_{N}\right)-\mathcal{S}_{N \tau}\left(\pi_{\tau}^{-} T_{N \tau}^{\diamond}\right) & 0 \\
0 & \mathcal{L}_{N \tau}
\end{array}\right) D \mathcal{G}_{N \tau}\left(U_{N \tau}^{\diamond}\right) \\
+\left(\begin{array}{cc}
D \mathcal{S}_{N \tau}\left(\pi_{\tau}^{-} \widetilde{T}_{N}\right)-D \mathcal{S}_{N \tau}\left(T_{N \tau}^{\diamond}\right) & 0 \\
0 & 0
\end{array}\right) \mathcal{G}_{N \tau}\left(U_{N \tau}^{\diamond}\right) \\
+\left(\begin{array}{cc}
\mathcal{S}_{N \tau}\left(\pi_{\tau}^{-} \widetilde{T}_{N}\right) & 0 \\
0 & \mathcal{L}_{N \tau}
\end{array}\right)\left(D \mathcal{G}_{N \tau}\left(\widetilde{U}_{N}\right)-D \mathcal{G}_{N \tau}\left(U_{N \tau}^{\diamond}\right)\right) \\
+\left(\begin{array}{cc}
D \mathcal{S}_{N \tau}\left(\pi_{\tau}^{-} \widetilde{T}_{N}\right) & 0 \\
0 & 0
\end{array}\right)\left(\mathcal{G}_{N \tau}\left(\widetilde{U}_{N}\right)-\mathcal{G}_{N \tau}\left(U_{N \tau}^{\diamond}\right)\right)
\end{aligned}
$$

We have to evaluate the quantities, for any $W_{N \tau}=\left(\boldsymbol{w}_{N \tau}, R_{N \tau}\right)$ in the unit sphere of $\mathbb{Z}_{N \tau}$, since the evaluating of the last two terms follow from Lemma 4.3 and an extension of it, we only consider the first two terms. All constants $c$ in what follows only depend on the norms $\left\|U_{N \tau}^{\diamond}\right\|_{\mathbb{Z}},\left\|\widetilde{U}_{N}\right\|_{\mathbb{Z}}$ and $\|\nu\|_{W^{2, \infty}(\mathbb{R})}$.

1) We have

$$
\left(\begin{array}{cc}
\mathcal{S}_{N \tau}\left(\pi_{\tau}^{-} \widetilde{T}_{N}\right)-\mathcal{S}_{N \tau}\left(\pi_{\tau}^{-} T_{N \tau}^{\diamond}\right) & 0 \\
0 & \mathcal{L}_{N \tau}
\end{array}\right) D \mathcal{G}_{N \tau}\left(U_{N \tau}^{\diamond}\right) W_{N \tau}=\left(\begin{array}{cc}
\mathcal{S}_{N \tau}\left(\pi_{\tau}^{-} T_{N \tau}^{\diamond}\right) & 0 \\
0 & \mathcal{L}_{N \tau}
\end{array}\right)\left(\begin{array}{c}
\left(A_{N \tau}, 0\right), \\
0
\end{array}\right)
$$

with

$$
\left.\left.A_{N \tau}=\operatorname{div}\left(\nu\left(T_{N \tau}^{\diamond}\right)-\nu\left(\widetilde{T}_{N}\right)\right) \nabla \mathcal{S}_{N \tau}\left(\pi_{\tau}^{-} \widetilde{T}_{N}\right)\right)\left(\left(\boldsymbol{u}_{N \tau}^{\diamond} \cdot \nabla\right) \boldsymbol{w}_{N \tau}+\left(\boldsymbol{w}_{N \tau} \cdot \nabla\right) \boldsymbol{u}_{N \tau}^{\diamond}\right)\right)
$$

There exists a constant $c$ only depending on the Lipschitz property of $\nu$ such that,

$$
\left\|\left(\begin{array}{cc}
\mathcal{S}_{N \tau}\left(\pi_{\tau}^{-} \widetilde{T}_{N}\right)-\mathcal{S}_{N \tau}\left(\pi_{\tau}^{-} T_{N \tau}^{\diamond}\right) & 0 \\
0 & 0
\end{array}\right) D \mathcal{G}_{N \tau}\left(U_{N \tau}^{\diamond}\right) W_{N \tau}\right\|_{\mathbb{Z}} \leqslant c\left\|T_{N \tau}^{\diamond}-\widetilde{T}_{N}\right\|_{L^{2}(\Omega)}\left\|\boldsymbol{w}_{N \tau}\right\|_{L^{2}(\Omega)^{d}}
$$

2) On the other hand, combing the second part of (4.43)

$$
\left\|\left(\begin{array}{cc}
D \mathcal{S}_{N \tau}\left(\pi_{\tau}^{-} \widetilde{T}_{N}\right)-\mathcal{S}_{N \tau}\left(T_{N \tau}^{\diamond}\right) & 0 \\
0 & 0
\end{array}\right) \mathcal{G}_{N \tau}\left(U_{N \tau}^{\diamond}\right)\right\|_{\mathbb{Z}} \leqslant c\left\|\widetilde{T}_{N}-T_{N}^{\diamond}\right\|_{L^{2}(\Omega)}\left\|R_{N \tau}\right\|_{L^{2}(\Omega)} .
$$

Lemma 4.7 Assume that $\nu$ is of class $\mathscr{C}^{2}$ on $\mathbb{R}$ and that the solution $(\boldsymbol{u}, p, T)$ of problem $(2.5)-(2.6)-(2.7)$ belong to $H^{2}\left(0, \widetilde{T} ; H^{s}(\Omega)^{d}\right) \times H^{2}\left(0, \widetilde{T} ; H^{s-1}(\Omega)\right) \times H^{2}\left(0, \widetilde{T} ; H^{s}(\Omega)\right)$ for a real number $s, s \geqslant 1$, and the data $\left(\boldsymbol{f}, T_{b}\right)$ belong to $L^{2}\left(0, \widetilde{T} ; H^{\sigma}(\Omega)^{d}\right) \times H^{2}\left(0, \widetilde{T} ; H^{\sigma+\frac{1}{2}}(\partial \Omega)\right)$ for a real number $\sigma$, $\sigma>\frac{d}{2}$. Then, the following estimate is satisfied

$$
\begin{array}{r}
\left\|\mathcal{F}_{N \tau}\left(U_{N \tau}^{\diamond}\right)\right\|_{\mathbb{Z}} \leqslant c\left(\left(|\tau|+N^{1-s}\right)\left(\|\boldsymbol{u}\|_{H^{2}\left(0, \widetilde{T} ; H^{s}(\Omega)^{d}\right)}+\|T\|_{H^{2}\left(0, \widetilde{T} ; H^{s}(\Omega)\right)}\right)+N^{1-s}\|p\|_{H^{2}\left(0, \widetilde{T} ; H^{s-1}(\Omega)\right)}\right) \\
+c^{\prime}\left(\left(|\tau|+N^{-\sigma}\right)\left(\|\boldsymbol{f}\|_{L^{2}\left(0, \widetilde{T} ; H^{\sigma}(\Omega)^{d}\right)}+\left\|T_{b}\right\|_{H^{2}\left(0, \widetilde{T} ; H^{\left.\sigma+\frac{1}{2}(\partial \Omega)\right)}\right.}\right)\right) .
\end{array}
$$

Proof: Since $\mathcal{F}(U)$ is zero, we use the triangle inequality

$$
\begin{aligned}
\left\|\mathcal{F}_{N \tau}\left(U_{N \tau}^{\diamond}\right)\right\|_{\mathbb{Z}} \leqslant\left\|U-U_{N \tau}^{\diamond}\right\|_{\mathbb{Z}}+\left\|\left(\begin{array}{cc}
\mathcal{S}(T)-\mathcal{S}_{N \tau}\left(\pi_{\tau}^{-} T_{N \tau}^{\diamond}\right) & 0 \\
0 & \mathcal{L}-\mathcal{L}_{N \tau}
\end{array}\right) \mathcal{G}(U)\right\|_{\mathbb{Z}} \\
+\left\|\left(\begin{array}{cc}
\mathcal{S}_{N \tau}\left(\pi_{\tau}^{-} T_{N \tau}^{\diamond}\right) & 0 \\
0 & \mathcal{L}_{N \tau}
\end{array}\right)\left(\mathcal{G}(U)-\mathcal{G}\left(U_{N \tau}^{\diamond}\right)\right)\right\|_{\mathbb{Z}} \\
\quad+\left\|\left(\begin{array}{cc}
\mathcal{S}_{N \tau}\left(\pi_{\tau}^{-} T_{N \tau}^{\diamond}\right) & 0 \\
0 & \mathcal{L}_{N \tau}
\end{array}\right)\left(\mathcal{G}\left(U_{N \tau}^{\diamond}\right)-\mathcal{G}_{N \tau}\left(U_{N \tau}^{\diamond}\right)\right)\right\|_{\mathbb{Z}}
\end{aligned}
$$


The first term is bounded in (4.38). Evaluating the second term follows from the continuity of the operator $\mathcal{S}_{N \tau}(\theta),(4.38),(4.22),(4.32),(4.16),(4.25),(4.35)$ and (4.20) by noting that: If $\left(\boldsymbol{F}, \boldsymbol{u}_{0}\right)$ denotes the first component of $\mathcal{G}(U), \mathcal{S}\left(\boldsymbol{F}, \boldsymbol{u}_{0}\right)$ is equal to $\boldsymbol{u}$ and $\boldsymbol{F}$ is equal to $\partial_{t} \boldsymbol{u}-\operatorname{div}(\nu(T) \nabla \boldsymbol{u})+$ $\operatorname{grad} p$. To bound the third one, we apply (4.31), (4.34) and by using triangle inequalities and estimate (4.38). Finally, proving the estimate for the fourth term is obtained from (4.31), (4.34) and by using the standard arguments for the error issued from numerical integration combined with the same arguments of the proof of Lemma 4.3 .

\subsection{The conclusive a priori error estimates}

Owing to Lemmas 4.5 to 4.7, all the assumptions needed to apply the theorem of Brezzi, Rappaz and Raviart [7, Thm 1] (see also [11, Chap. IV, Thm 3.1]) are satisfied.

This leads to the main result of this section.

Theorem 4.8 Let $(\boldsymbol{u}, p, T)$ be a solution of problem (2.5)-(2.6)-(2.7) which satisfies Assumption 4.4 and belongs to $H^{2}\left(0, \widetilde{T} ; H^{s}(\Omega)^{d}\right) \times H^{2}\left(0, \widetilde{T} ; H^{s-1}(\Omega)\right) \times H^{2}\left(0, \widetilde{T} ; H^{s}(\Omega)\right), s>1$. We moreover assume that the function $\nu$ is of class $\mathscr{C}^{2}$ on $\mathbb{R}$ with bounded derivatives and that the data $\left(\boldsymbol{f}, T_{b}\right)$ belong to $L^{2}\left(0, \widetilde{T} ; H^{\sigma}(\Omega)^{d}\right) \times H^{2}\left(0, \widetilde{T} ; H^{\sigma+\frac{1}{2}}(\partial \Omega)\right)$, for a real number $\sigma, \sigma>\frac{d}{2}$. Then, there exist a neighbourhood of $(\boldsymbol{u}, T)$ in $\mathbb{Z}$ and positive real numbers $\tau_{0}$ and $N_{0}$ such that, for all $\tau,|\tau| \leqslant \tau_{0}$ and for all $N \geqslant N_{0}$, problem (3.13)-(3.14)-(3.15) has a unique solution $\left(\boldsymbol{u}_{N \tau}, p_{N \tau}, T_{N \tau}\right)$ in this neighbourhood. Moreover, this solution satisfies the following a priori error estimate

$$
\begin{gathered}
\left\|\boldsymbol{u}-\boldsymbol{u}_{N \tau}\right\|_{L^{2}\left(0, \widetilde{T} ; H^{1}(\Omega)^{d}\right)}+\left\|T-T_{N \tau}\right\|_{L^{2}\left(0, \widetilde{T} ; H^{1}(\Omega)\right)}+N^{-(d-1) / 2}\left\|p-p_{N \tau}\right\|_{L^{2}\left(0, \widetilde{T} ; L^{2}(\Omega)\right)} \\
\leqslant c\left(|\tau|+N^{1-s}\right) c(\boldsymbol{u}, p, T)+c^{\prime}\left(|\tau|+N^{-\sigma}\right) c\left(\boldsymbol{f}, T_{b}\right) .
\end{gathered}
$$

where the constant $c(\boldsymbol{u}, p, T)$ only depends on the solution $(\boldsymbol{u}, p, T)$ of problem (2.5)-(2.6)-(2.7) and $c\left(\boldsymbol{f}, T_{b}\right)$ only depends on the data $\boldsymbol{f}$ and $T_{b}$.

This estimate is fully optimal. Moreover Assumption 4.4 is not restrictive, it only implies the local uniqueness of the solution $(\boldsymbol{u}, p, T)$ which seems likely. 


\section{$5 \quad$ Numerical experiments}

In order to confirm these results numerically, we made several experiments by using the code MATLAB software. The numerical experiments have been performed in the two-dimensional case, on the square $\Omega=]-1,1\left[^{2}\right.$. The results are obtained using a $\mathbb{P}_{N}^{0}(\Omega)^{d} \times\left(\mathbb{P}_{N-2}(\Omega) \cap L_{\circ}^{2}(\Omega)\right) \times \mathbb{P}_{N}(\Omega)$ space discretisation of $(\boldsymbol{u}, p, T)$, and the backward Euler discretization with uniform time step $\tau_{j}=\delta t$. Finally, at each step, linear systems are obtained and solved using as preconditionners an incomplete factorization of type Cholesky associated with the preconditioned conjugate gradient method (we can also use an incomplete $L U$ factorization associated with the Gmres method or bicgstab method).

\subsection{Convergence in time}

In the first case we test the convergence of our code where the Navier-Stokes equations are independent of the temperature. It means taking $\nu(T)=1$ first and $\nu(T)(x, y)=x y t+1$, second, then comparing the two tests with the case where the problem is coupled with $\nu(T)=\sqrt{T^{2}+1}+2$. The exact solution is given by

$$
\begin{gathered}
\boldsymbol{u}(x, y)=\left(\begin{array}{c}
\sin ((\pi+t) y) \cos ((\pi+t) x) \\
-\cos ((\pi+t) y) \sin ((\pi+t) x)
\end{array}\right), \\
p(x, y)=\sin ((\pi+t) x) \cos ((\pi+t) y), \quad T(x, y)=t \sin (x+y) .
\end{gathered}
$$

We note that this solution satisfies the condition of incompressibility. We present in the following Tables 1, 2 and 3 the convergence towards the solution (5.1)-(5.2) as a function of $\delta t$ with the viscosity $\nu$ is a constant (Table 1), a function dependent of the space variable (Table 2) and a function which depends on the temperature $T$ (Table 3 ). All the tests in this subsection are computed for $N=15$ at $\widetilde{T}=0.1$.

\begin{tabular}{|c|c|c|c|c|}
\hline$\delta t$ & $10^{-1}$ & $5.10^{-2}$ & $10^{-2}$ & $5.10^{-3}$ \\
\hline \hline$\left\|\boldsymbol{u}-\boldsymbol{u}_{N \tau}\right\|_{L^{2}(\Omega)^{2}}$ & $5.7418 \times 10^{-4}$ & $3.1626 \times 10^{-4}$ & $6.7873 \times 10^{-5}$ & $3.4176 \times 10^{-5}$ \\
\hline$\left\|\boldsymbol{u}-\boldsymbol{u}_{N \tau}\right\|_{H^{1}(\Omega)^{2}}$ & $0.042 \times 10^{-3}$ & $0.023 \times 10^{-3}$ & $4.8310 \times 10^{-4}$ & $2.4293 \times 10^{-4}$ \\
\hline$\left\|p-p_{N \tau}\right\|_{L^{2}(\Omega)}$ & $5.010 \times 10^{-3}$ & $0.255 \times 10^{-3}$ & $0.051 \times 10^{-3}$ & $0.025 \times 10^{-3}$ \\
\hline$\left\|T-T_{N \tau}\right\|_{L^{2}(\Omega)}$ & $0.029 \times 10^{-3}$ & $0.016 \times 10^{-3}$ & $3.6463 \times 10^{-4}$ & $1.8486 \times 10^{-4}$ \\
\hline
\end{tabular}

\begin{tabular}{|c|c|c|c|}
\hline$\delta t$ & $10^{-3}$ & $5.10^{-4}$ & $10^{-4}$ \\
\hline \hline$\left\|\boldsymbol{u}-\boldsymbol{u}_{N \tau}\right\|_{L^{2}(\Omega)^{2}}$ & $6.8703 \times 10^{-6}$ & $3.4373 \times 10^{-6}$ & $8.1224 \times 10^{-7}$ \\
\hline$\left\|\boldsymbol{u}-\boldsymbol{u}_{N \tau}\right\|_{H^{1}(\Omega)^{2}}$ & $4.8781 \times 10^{-5}$ & $2.4405 \times 10^{-5}$ & $4.5312 \times 10^{-6}$ \\
\hline$\left\|p-p_{N \tau}\right\|_{L^{2}(\Omega)}$ & $5.0445 \times 10^{-4}$ & $2.5223 \times 10^{-4}$ & $5.0214 \times 10^{-5}$ \\
\hline$\left\|T-T_{N \tau}\right\|_{L^{2}(\Omega)}$ & $3.7387 \times 10^{-5}$ & $1.8720 \times 10^{-5}$ & $4.0220 \times 10^{-6}$ \\
\hline
\end{tabular}

Table 1: Convergence to the solution (5.1)-(5.2) for $\nu(T)=1$. 


\begin{tabular}{|c|c|c|c|c|}
\hline$\delta t$ & $10^{-1}$ & $5.10^{-2}$ & $10^{-2}$ & $5.10^{-3}$ \\
\hline \hline$\left\|\boldsymbol{u}_{-} \boldsymbol{u}_{N \tau}\right\|_{L^{2}(\Omega)^{2}}$ & $5.3973 \times 10^{-4}$ & $3.6949 \times 10^{-4}$ & $9.3931 \times 10^{-5}$ & $4.8388 \times 10^{-5}$ \\
\hline$\left\|\boldsymbol{u}-\boldsymbol{u}_{N \tau}\right\|_{H^{1}(\Omega)^{2}}$ & $0.026 \times 10^{-3}$ & $0.017 \times 10^{-3}$ & $4.1788 \times 10^{-4}$ & $2.1451 \times 10^{-4}$ \\
\hline$\left\|p-p_{N \tau}\right\|_{L^{2}(\Omega)}$ & $5.009 \times 10^{-3}$ & $0.235 \times 10^{-3}$ & $0.052 \times 10^{-3}$ & $0.026 \times 10^{-3}$ \\
\hline$\left\|T-T_{N \tau}\right\|_{L^{2}(\Omega)}$ & $0.029 \times 10^{-3}$ & $0.016 \times 10^{-3}$ & $3.6445 \times 10^{-4}$ & $1.8477 \times 10^{-4}$ \\
\hline
\end{tabular}

\begin{tabular}{|c|c|c|c|}
\hline$\delta t$ & $10^{-3}$ & $5.10^{-4}$ & $10^{-4}$ \\
\hline \hline$\left\|\boldsymbol{u}_{-} \boldsymbol{u}_{N \tau}\right\|_{L^{2}(\Omega)^{2}}$ & $9.9118 \times 10^{-6}$ & $4.9707 \times 10^{-6}$ & $1.0127 \times 10^{-6}$ \\
\hline$\left\|\boldsymbol{u}-\boldsymbol{u}_{N \tau}\right\|_{H^{1}(\Omega)^{2}}$ & $4.3817 \times 10^{-5}$ & $2.1966 \times 10^{-5}$ & $5.1209 \times 10^{-6}$ \\
\hline$\left\|p-p_{N \tau}\right\|_{L^{2}(\Omega)}$ & $5.1951 \times 10^{-4}$ & $2.5988 \times 10^{-5}$ & $5.6759 \times 10^{-5}$ \\
\hline$\left\|T-T_{N \tau}\right\|_{L^{2}(\Omega)}$ & $3.7367 \times 10^{-5}$ & $1.8709 \times 10^{-5}$ & $3.7460 \times 10^{-6}$ \\
\hline
\end{tabular}

Table 2: Convergence to the solution $(5.1)-(5.2)$ for $\nu(T)(x, y)=x y t+1$.

\begin{tabular}{|c|c|c|c|c|}
\hline$\delta t$ & $10^{-1}$ & $5.10^{-2}$ & $10^{-2}$ & $5.10^{-3}$ \\
\hline \hline$\left\|\boldsymbol{u}-\boldsymbol{u}_{N \tau}\right\|_{L^{2}(\Omega)^{2}}$ & $5.7488 \times 10^{-4}$ & $3.1659 \times 10^{-4}$ & $2.2987 \times 10^{-4}$ & $3.4208 \times 10^{-5}$ \\
\hline$\left\|\boldsymbol{u}-\boldsymbol{u}_{N \tau}\right\|_{H^{1}(\Omega)^{2}}$ & $0.056 \times 10^{-3}$ & $0.034 \times 10^{-3}$ & $2 \times 10^{-3}$ & $2.4320 \times 10^{-4}$ \\
\hline$\left\|p-p_{N \tau}\right\|_{L^{2}(\Omega)}$ & $7.540 \times 10^{-3}$ & $0.345 \times 10^{-3}$ & $0.062 \times 10^{-3}$ & $0.039 \times 10^{-3}$ \\
\hline$\left\|T-T_{N \tau}\right\|_{L^{2}(\Omega)}$ & $0.059 \times 10^{-3}$ & $0.056 \times 10^{-3}$ & $2.6598 \times 10^{-4}$ & $1.9655 \times 10^{-4}$ \\
\hline
\end{tabular}

\begin{tabular}{|c|c|c|c|}
\hline$\delta t$ & $10^{-3}$ & $5.10^{-4}$ & $10^{-4}$ \\
\hline \hline$\left\|\boldsymbol{u}-\boldsymbol{u}_{N \tau}\right\|_{L^{2}(\Omega)^{2}}$ & $6.8766 \times 10^{-5}$ & $3.4723 \times 10^{-6}$ & $1.0015 \times 10^{-5}$ \\
\hline$\left\|\boldsymbol{u}-\boldsymbol{u}_{N \tau}\right\|_{H^{1}(\Omega)^{2}}$ & $4.8835 \times 10^{-5}$ & $2.5093 \times 10^{-5}$ & $3.8159 \times 10^{-6}$ \\
\hline$\left\|p-p_{N \tau}\right\|_{L^{2}(\Omega)}$ & $5.1045 \times 10^{-4}$ & $2.5247 \times 10^{-4}$ & $4.5284 \times 10^{-5}$ \\
\hline$\left\|T-T_{N \tau}\right\|_{L^{2}(\Omega)}$ & $3.9524 \times 10^{-5}$ & $1.8720 \times 10^{-5}$ & $3.1209 \times 10^{-6}$ \\
\hline
\end{tabular}

Table 3: Convergence to the solution $(5.1)-(5.2)$ for $\nu(T)=\sqrt{T^{2}+1}+2$.

In Figure 1 we use the same results as in Tables 1, 2 and 3, to present the quantities :

$\log _{10}\left\|\boldsymbol{u}-\boldsymbol{u}_{N \tau}\right\|_{L^{2}(\Omega)^{2}}, \log _{10}\left\|\boldsymbol{u}-\boldsymbol{u}_{N \tau}\right\|_{H^{1}(\Omega)^{2}}, \log _{10}\left\|p-p_{N \tau}\right\|_{L^{2}(\Omega)}$, and $\log _{10}\left\|T-T_{N \tau}\right\|_{L^{2}(\Omega)}$

as a function of $\log _{10}(\delta t)$ for $N=15$ at time $\widetilde{T}=0.1$.

We note that the error of the velocity, pressure and temperature estimates are very close when taking $\nu$ a constant, or a function dependent of the space variable, or a function that depends on the temperature $T$ which shows the effectiveness of the code developped for the coupled problem. 

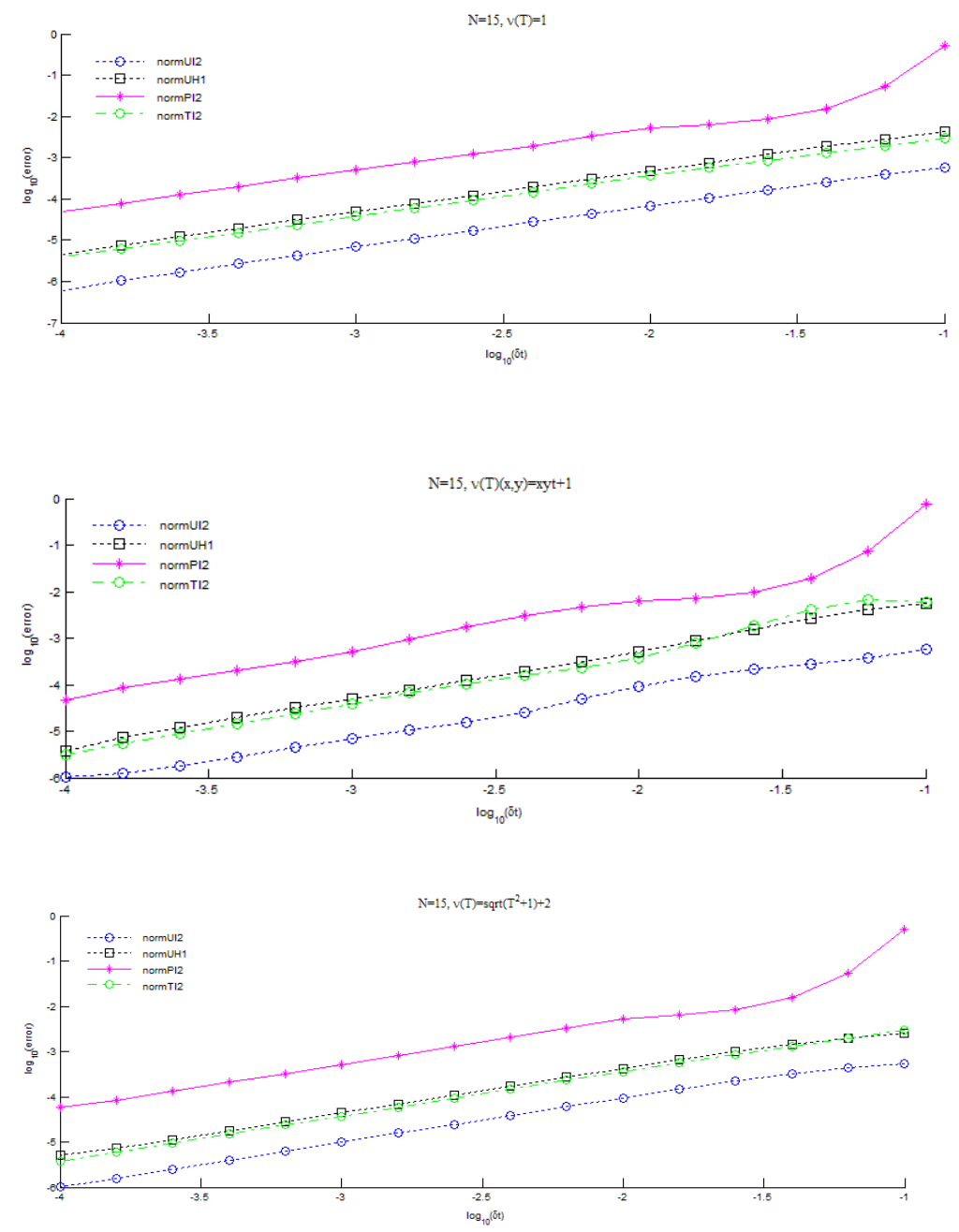

Figure 1: The errors for the solution (5.1)-(5.2)

We give in this table 4 the relative residual and the iteration number of the preconditioned conjugate gradient for the solution (5.1)-(5.2), the first is for $\nu(T)=\sqrt{T^{2}+1}+2$ and the second for $\nu(T)=1$.

\begin{tabular}{|c|c|c|c|c|}
\hline$\delta t$ & $10^{-1}$ & $10^{-2}$ & $10^{-3}$ & $10^{-4}$ \\
\hline \hline Res & $\begin{array}{c}1.05 \\
\times 10^{-9}\end{array}$ & $\begin{array}{c}4.86 \\
\times 10^{-9}\end{array}$ & $\begin{array}{c}4.078 \\
\times 10^{-8}\end{array}$ & $\begin{array}{c}1.02 \\
\times 10^{-7}\end{array}$ \\
\hline iter & 73 & 92 & 98 & 102 \\
\hline
\end{tabular}

\begin{tabular}{|c|c|c|c|c|}
\hline$\delta t$ & $10^{-1}$ & $10^{-2}$ & $10^{-3}$ & $10^{-4}$ \\
\hline \hline Res & $\begin{array}{c}8.95 \\
\times 10^{-11}\end{array}$ & $\begin{array}{c}2.26 \\
\times 10^{-10}\end{array}$ & $\begin{array}{c}1.56 \\
\times 10^{-8}\end{array}$ & $\begin{array}{c}5.35 \\
\times 10^{-7}\end{array}$ \\
\hline iter & 82 & 90 & 95 & 99 \\
\hline
\end{tabular}

Table 4: The residual and number of iterations for $\nu(T)=\sqrt{T^{2}+1}+2$ and $\nu(T)=1$. 


\subsection{Convergence in space}

For the second numerical experiment we work with the solution given by

$$
\begin{gathered}
\boldsymbol{u}(x, y)=\left(\begin{array}{c}
t\left(1-x^{2}\right)^{\frac{5}{2}} y\left(1-y^{2}\right)^{\frac{3}{2}} \\
-t\left(1-x^{2}\right)^{\frac{3}{2}} x\left(1-y^{2}\right)^{\frac{5}{2}}
\end{array}\right), \\
p(x, y)=\sin (x y t), \quad T(x, y)=x^{2}+y^{2}+t .
\end{gathered}
$$

In Figure 2 we present the quantities:

$$
\begin{gathered}
\log _{10}\left\|\boldsymbol{u}-\boldsymbol{u}_{N \tau}\right\|_{L^{2}(\Omega)^{2}}, \log _{10}\left\|\boldsymbol{u}-\boldsymbol{u}_{N \tau}\right\|_{H^{1}(\Omega)^{2}}, \log _{10}\left\|p-p_{N \tau}\right\|_{L^{2}(\Omega)}, \\
\log _{10}\left\|T-T_{N \tau}\right\|_{L^{2}(\Omega)} \quad \text { and } \quad \log _{10}\left\|T-T_{N \tau}\right\|_{H^{1}(\Omega)}
\end{gathered}
$$

as function of $N$, for $N$ varing from 5 to 35 in both cases by considering $\delta t=10^{-2}, \nu(T)=\frac{1}{T^{2}+1}$ at $\widetilde{T}=0.1$. In Figure 3 we present the same quantities with $\delta t=10^{-2}, \nu(T)=\frac{1}{T^{2}+1}$ but at $\widetilde{T}=1$.

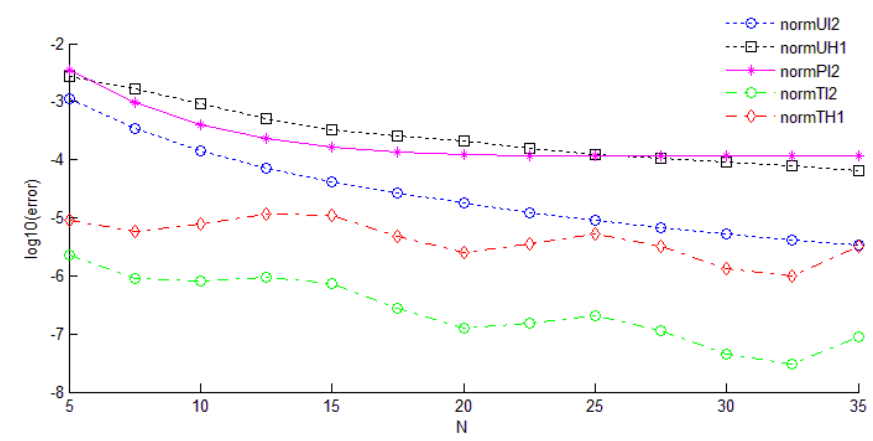

Figure 2: The estimations of error of the solution (5.3)-(5.4)

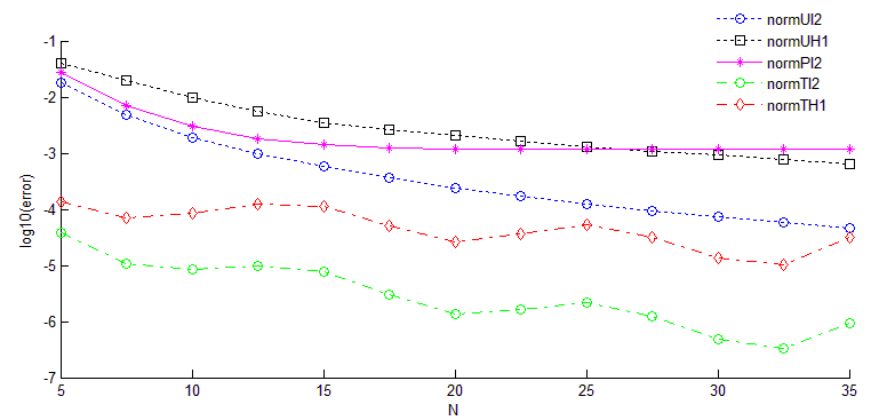

Figure 3: The estimations of error of the solution (5.3)-(5.4)

By letting $N$ go through 5 to 40 , we note that the error of norms $\left\|\boldsymbol{u}-\boldsymbol{u}_{N \tau}\right\|_{L^{2}(\Omega)^{2}},\left\|\boldsymbol{u}-\boldsymbol{u}_{N \tau}\right\|_{H^{1}(\Omega)^{2}}$ and $\left\|p-p_{N \tau}\right\|_{L^{2}(\Omega)}$ decrease until $N=35$ and stagnated for $N>35$. The error norms $\left\|T-T_{N \tau}\right\|_{L^{2}(\Omega)}$ and $\left\|T-T_{N \tau}\right\|_{H^{1}(\Omega)}$ decrease but in a sinusoidale way. 


\subsection{A further computation}

- We consider the exact solution given by

$$
\begin{gathered}
\boldsymbol{u}(x, y)=\left(\begin{array}{c}
t\left(1-x^{2}\right)^{5 / 2} y\left(1-y^{2}\right)^{3 / 2} \\
-t\left(1-x^{2}\right)^{3 / 2} x\left(1-y^{2}\right)^{5 / 2}
\end{array}\right) \\
p(x, y)=\sin (x y t), \quad T(x, y)=t x+y
\end{gathered}
$$

We present the curves of isovalues of the two components of the velocity, of the temperature and of the pressure of the exact solution in Figure 4 and of the discrete solution in Figure 5 for $\nu(T)=10^{-5}(T+1)$ with $N=40, \delta t=10^{-3}$ at $\widetilde{T}=0.1$. We see a very high resemblance between the exact solution and the discrete solution. This shows the efficiency of our preconditionner.
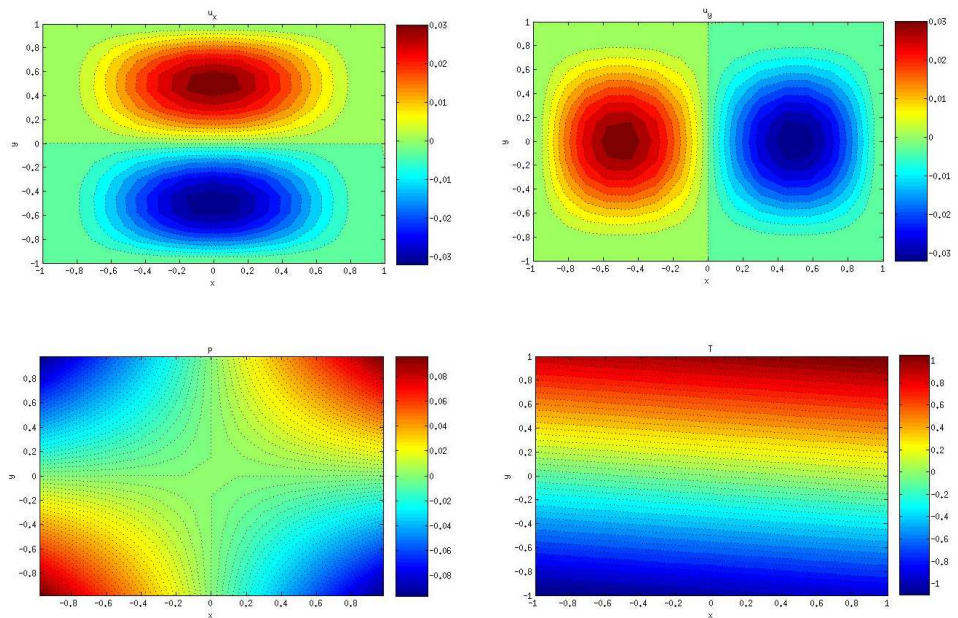

Figure 4: The isovalues of the two components of the exact velocity (on the top), of the exact temperature and of the exact pressure (on the bottom) obtained with $N=40$.
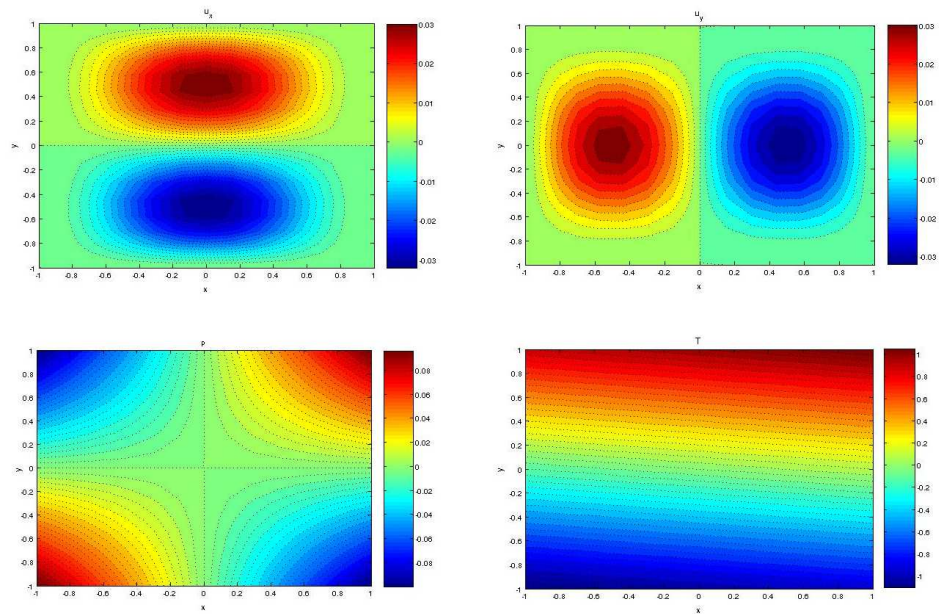

Figure 5: The isovalues of the two components of the discrete velocity (on the top), of the discrete temperature and of the discrete pressure (on the bottom) obtained with $N=40$. 
- We present now numerical experiments where $\nu(T)$ is taken equal to $T+1$ when the exact solution is unknown. We work with the data $\boldsymbol{f}=\left(f_{x}, f_{y}\right)$ and $g$ given by

$$
\boldsymbol{f}(x, y)=\left(\begin{array}{c}
x^{2}+y^{2} t \\
x^{2}+y^{2}+t^{2}
\end{array}\right), \quad g(x, y)=\sin (x y t) .
$$

The boundary condition is replaced by

$$
\boldsymbol{u}=\boldsymbol{h} \quad \text { on } \quad \partial \Omega,
$$

the boundary temperature $T_{b}$ and velocity $\boldsymbol{h}=\left(h_{1}, h_{2}\right)$ being given by

$$
\left\{\begin{array}{c}
h_{1}(x, y)=y t, h_{2}(x, y)=y^{2} t, \quad T_{b}(x, y)=-y+t+1 \quad \text { if } x=-1, \\
h_{1}(x, y)=y t, h_{2}(x, y)=-y^{2} t, \quad T_{b}(x, y)=y+t+1 \quad \text { if } x=1, \\
h_{1}(x, y)=-x^{2} t, h_{2}(x, y)=-x t, \quad T_{b}(x, y)=-x^{3}+t+1 \quad \text { if } y=-1, \\
h_{1}(x, y)=x^{2} t, h_{2}(x, y)=-x t, \quad T_{b}(x, y)=x^{3}+t+1 \quad \text { if } y=1,
\end{array}\right.
$$

The initial velocity and temperature are taken as

$$
\boldsymbol{u}(t=0)=\mathbf{0} \quad \text { and } \quad T(t=0)=x^{3} y+1 .
$$

Note that the data satisfy the usual compatibility condition $\int_{\partial \Omega} \boldsymbol{h}(\tau) \cdot \boldsymbol{n}(\tau) d(\tau)=0$.

The discrete solution computed with $N=30, \delta t=10^{-2}$ at $\widetilde{T}=1$ is presented in Figure 6 . The two components of the velocity are presented in Figure 7, the pressure and temperature in Figure 8 with $N=25, \delta t=5 \cdot 10^{-2}$ at $\widetilde{T}=0.1$.
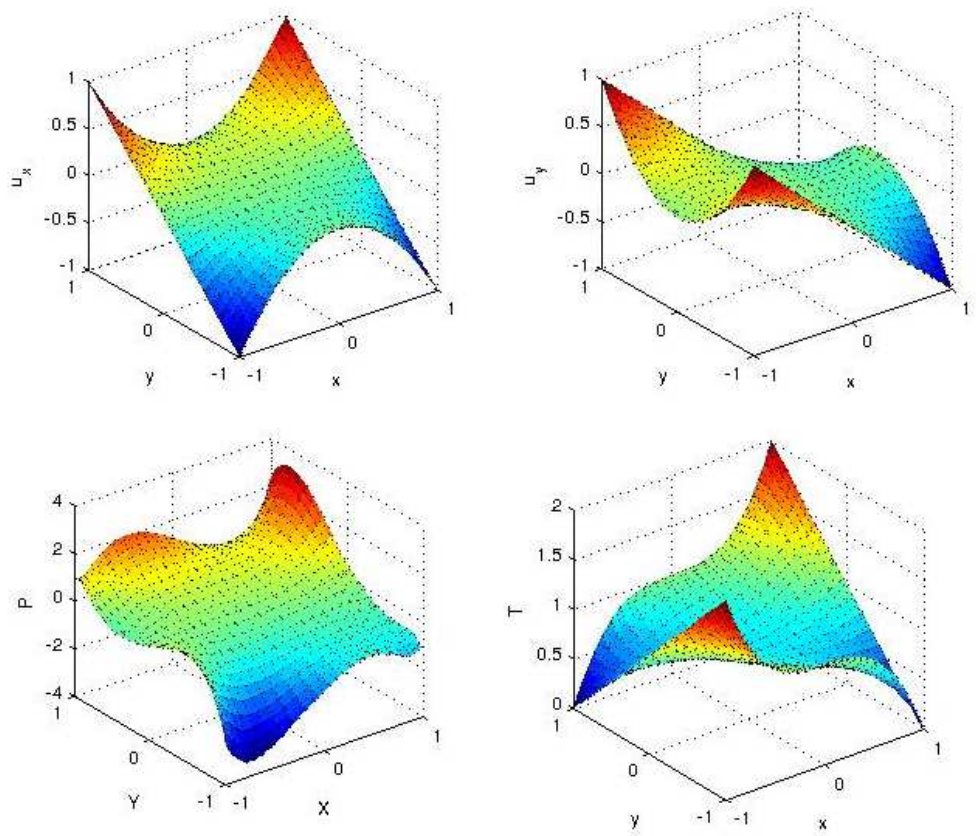

Figure 6: The discrete solution issued from (5.7)-(5.8) 

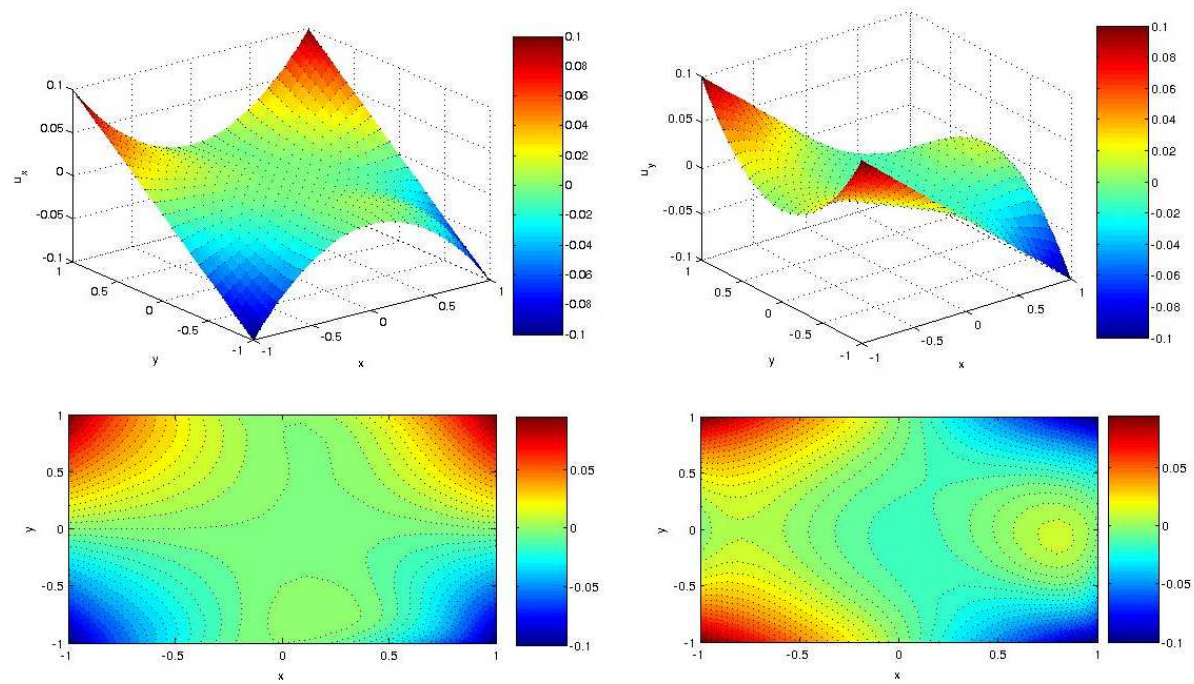

Figure 7: The values and the isovalues of the two components of the velocity issued from (5.7)-(5.8)
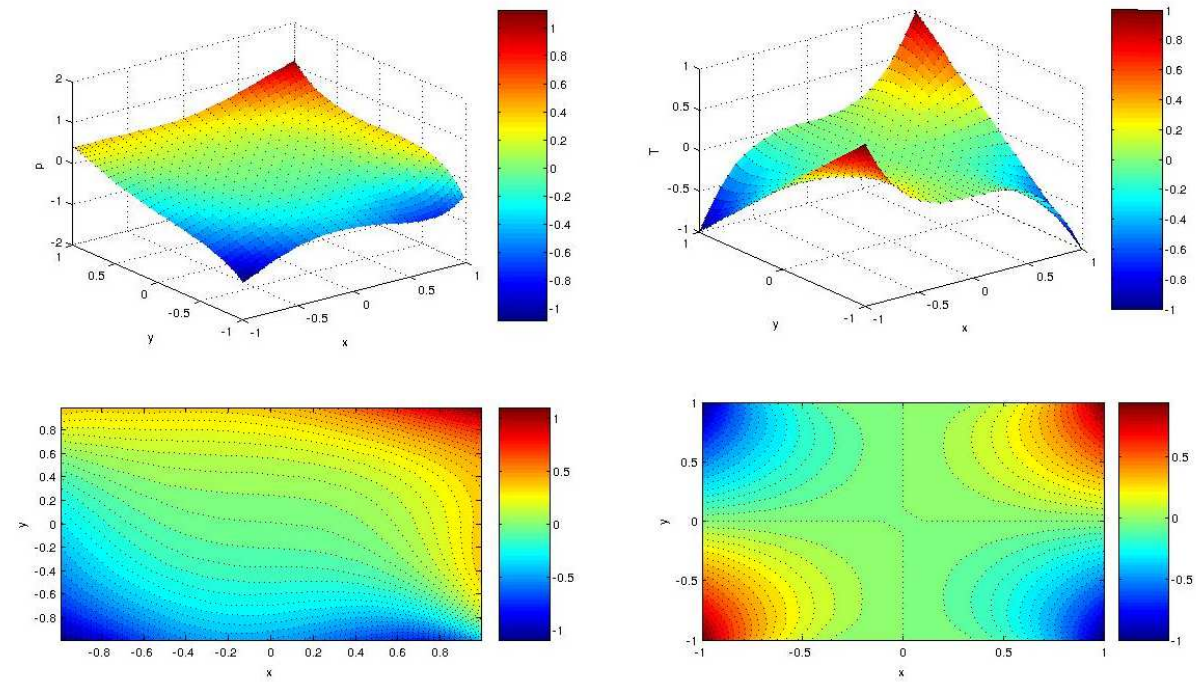

Figure 8: The values and the isovalues of the pressure and the temperature issued from (5.7)-(5.8)

All the tests of convergence confirm the theoretical estimate obtained in (4.47): the convergence in time of the backward Euler's schema is of order 1, the convergence in space is of spectral type. 


\section{References}

[1] R. Agroum, S. Aouadi, C. Bernardi, J. Satouri - Spectral discretization of the Navier-Stokes problem coupled with the heat equation, Internal Report, Laboratoire Jacques-Louis Lions (2013), submitted.

[2] K. Allali, F. Bikany, A. Taik, V. Volpert - Numerical simulations of heat explosion with convection in porous media, preprint arXiv:1309.2837 (2013).

[3] C. Bernardi, T. Chacón Rebollo, F. Hecht, R. Lewandowski - Automatic insertion of a turbulence model in the finite element discretization of the Navier-Stokes equations, Math. Models and Methods in Applied Sciences 19 (2009), 1139-1183.

[4] C. Bernardi, F. Coquel, P.-A. Raviart - Automatic coupling and finite element discretization of the Navier-Stokes and heat equations, Internal Report 10001, Laboratoire Jacques-Louis Lions (2010).

[5] C. Bernardi, Y. Maday - Spectral Methods, in the Handbook of Numerical Analysis, Vol V, P.G. Ciarlet and J.-L. Lions eds., North-Holland (1997), 209-485.

[6] C. Bernardi, Y, Maday, F. Rapetti - Discrétisations variationnelles de problèmes aux limites elliptiques, Collection "Mathématiques et Applications" 45, Springer-Verlag (2004).

[7] F. Brezzi, J. Rappaz, P.-A. Raviart - Finite dimensionnal approximation of nonlinear problems, Part I: Branches of nonsingular solutions, Numer. Math. 36 (1980), 1-25.

[8] M. Bulíček, E. Feireisl, J. Málek - A Navier-Stokes-Fourier system for incompressible fluids with temperature dependent material coefficients, Nonlinear Analysis: Real World Applications 10 (2009), 992-1015.

[9] D. Jerison, C. E. Kenig — The inhomogeneous Dirichlet problem in Lipschitz domains, J. Funct. Anal. 130 (1995), 161-219.

[10] V. Girault, P.-A. Raviart - Finite Element Approximation of the Navier-Stokes Equations, Lecture Notes in Mathematics 749, Springer-Verlag, Berlin (1979).

[11] V. Girault, P.-A. Raviart — Finite Element Methods for Navier-Stokes Equations. Theory and Algorithms, Springer-Verlag, Berlin (1986).

[12] M. Schatzman - Analyse numérique, InterEditions, Paris (1991).

[13] R. Temam - Navier-Stokes Equations. Theory and Numerical Analysis, Studies in Mathematics and its Applications, Vol. 2, North-Holland Publishing Co. (1977). 